\title{
jouvence, a new human H/ACA snoRNA involves in the control of cell proliferation and differentiation
}

Authors: Flaria El-Khoury ${ }^{1 \dagger}$, Jérôme Bignon ${ }^{2 \dagger}$, and Jean-René Martin ${ }^{1 *}$

(1) Equipe: Imagerie Cérébrale Fonctionnelle et Comportements (ICFC), Institut des Neurosciences Paris-Saclay (Neuro-PSI)

UMR-9197, CNRS/Université Paris-Saclay,

1 Avenue de la Terrasse (Bat. 32/33)

91198, Gif-sur-Yvette,

France

Tel: (33) 01.69.82.41.80 / Fax: (33) 01.69.82.34.47

(1*) corresponding author: Jean-René Martin: e-mail: jean-rene.martin@inaf.cnrs-gif.fr

$\dagger=$ equal first authors (these authors contribute equally to this work)

(2) Institut de Chimie des Substances Naturelles,

CNRS, Université Paris-Saclay,

Gif-sur-Yvette, France 


\begin{abstract}
Small nucleolar RNAs (snoRNAs) are non-coding RNAs conserved from archeobacteria to mammals. In humans, various snoRNAs have been associated with pathologies as well as with cancer. Recently in Drosophila, a new snoRNA named jouvence has been involved in lifespan. Since snoRNAs are well conserved through evolution, both structurally and functionally, jouvence orthologue has been identified in human, allowing hypothesizing that jouvence could display a similar function (increasing healthy lifespan) in human. Here, we report the characterization of the human snoRNA-jouvence, which was not yet annotated in the genome. We show, both in stably cancerous cell lines and in primary cells, that its overexpression stimulates the cell proliferation. In contrast, its knockdown, by siRNA leads to an opposite phenotype, a decrease in cell proliferation. Transcriptomic analysis reveals that overexpression of jouvence leads to a dedifferentiation signature of the cells, a cellular effect comparable to rejuvenation. Inversely, the knockdown of jouvence leads to a decrease of genes involved in ribosomes biogenesis and spliceosome in agreement with the canonical role of a H/ACA box snoRNA. In this context, jouvence could represent a now tool to fight against the deleterious effect of aging, as well as a new target in cancer therapy.
\end{abstract}

\title{
Introduction
}

Small nucleolar RNAs (snoRNAs), which consist of 60-300 nucleotides and conserved from archeobacteria to mammals, are non-coding RNAs localized in the nucleolus where they are associated with proteins to form small nucleolar RiboNucleoProtein (snoRNPs) $[1,2]$. They can be subdivided, based on conserved secondary structure and functional RNA motifs, in two major classes, the box C/D and the box H/ACA. They are predicted to guide nucleotide modifications mainly of ribosomal RNAs (rRNAs). Most box C/D performs the 2'-Omethylation of RNAs through fibrillarin, while the box H/ACA are known to convert the uridine to pseudouridine through dyskerin [1-4]. However, as more recently reported, H/ACA snoRNA seems to also pseudouridinylate other RNA substrates, such as mRNA and long-non-codingRNAs (lncRNAs) [5]. In addition, few other functions have also been assigned to H/ACA box snoRNAs, as for instance chromatin remodelling [5]. In humans, some pathologies have been associated with various H/ACA snoRNAs, as the congenital dyskeratose, in which patients have shorter telomeres [6,7], while the snoRNA HBII-52, a human C/D box, has been involved in the alternative splicing of the serotonine receptor $2 \mathrm{C}[8,9]$. 
In addition to their reported role in some specific pathologies, recent reports suggest that snoRNAs have also tumor-suppressive or oncogenic functions in various cancer types [10]. Indeed, several snoRNAs have been reported to participate in many biological cancer processes, as inactivation of growth suppressors and cell death, activation of invasion and metastasis, as well as sustained proliferative signalling [11]. Moreover, some evidences suggest that snoRNA could also play a role in cancer stem cells (CSC), as these last have the capacities of selfrenewal, differentiation and tumorigenicity [12]. Therefore, in this context, snoRNAs could have potential applications for cancer diagnosis and therapy.

In the last few years, in Drosophila, we have identified a new H/ACA box small nucleolar RNA (snoRNA) (a non-coding RNA), named jouvence (jou), the deletion of which reduces lifespan [13]. Inversely, its overexpression increases lifespan. In Drosophila, jouvence is required in the epithelium of the gut, and more precisely in enterocytes, while its deletion yields to the gut hyperplasia/dysplasia in aged flies [13]. Since the snoRNAs are well conserved throughout evolution, both structurally and functionally [1-4], we have identified its orthologues in mouse and human. The mouse genome contains two snoRNAs jouvence genes, whereas only one copy has been identified in human, located on chromosome 11. RT-PCR show that, both in mouse and human, jouvence orthologues are expressed suggesting that they might be functional [13]. Thus, these results allow to hypothesize that jouvence could display a similar function (increasing healthy lifespan) in mammals, including human.

Here, we have characterized the newly identified snoRNA-jouvence in human, which was not yet annotated. First, we have defined its genomic map as well as its predicted secondary structure. Second, we have shown, by RT-qPCR, that several cell types expressed jouvence. We have also shown that its overexpression importantly stimulates the proliferation of the cells, both in immortalized cancerous cells lines, as HCT116 and Caco-2, as well as on non-cancerous cell lines as HEK293 and RPE1, and even in primary cells as HUVEC. Inversely, its knockdown, by transitory transfection with siRNA leads to an opposite phenotype, inhibiting the proliferation of the cells. In a step further, a transcriptomic analysis (RNA-Seq) performed on HCT116 overexpressing cells reveals a signature of dedifferentiation of the cells, compatible with a rejuvenation. Finally, a similar transcriptomic analysis performed on the cells in which jouvence is knocked-down by siRNA reveals a typical and canonical snoRNA phenotype, meaning a strong decrease of the ribosome biogenesis as well as a decrease of the spliceosome pathways. Therefore, since jouvence depletion reduces cancer cell proliferation, we hypothesis that it could represent a good candidate to fight against cancer. 


\section{Results}

\section{Genomic map, structure, and localization of the human snoRNA-jouvence.}

Based on the tertiary (3D) structure of the Drosophila snoRNA-jouvence through the uses of the infernal software, we have identified the orthologue of jouvence in mouse and human, which was not yet annotated (13]. Human jouvence (shortly named $h$-jou) is located on the chromosome 11 (GRCh38.p12) in a long intron of the TEA domain family member 1 gene (named TEAD1) (Figure 1A). The human genome contains a single copy of jouvence, while two copies have been identified in the mouse genome (for the human sequence of jouvence, see Figure 10 in Soulé et al., 2020) [13]. Since jouvence possesses a primary sequence corresponding to a H/ACA box, its secondary structure has been deduced from the UNAFold software, and as expected is folded to form a double hairpin (Figure 1B), a typical structure of the H/ACA box snoRNA [1,2]. In a step further, the expression level of jouvence was determined by RT-qPCR, in some well-established human cell lines (Figure 1C). The snoRNA is weakly detected in the nine tested cell lines (first $\mathrm{Ct}$ around 30 to 33). Expression levels were close for all the cell lines, with HEK293 cells expressing a little bit more jouvence compared to the other cell lines, with a dCt (Ct snoRNA - Ct reference gene GAPDH) around 10. The U87MG glioblastoma cells had the lowest expression level with a dCt around 15.

\section{The overexpression of $h$-jou stimulates the proliferation of the cells.}

In order to determine the effect of the $h$-jou on human cell lines, the human jouvence (159 bp) was stably transfected into HCT116, Caco2 and HEK293 cell lines respectively. The proliferation of these cells was compared to the corresponding vector (empty-plasmid) stably transfected cells. The proliferation was conducted for a period of nearly one week for the different cell lines. The cell counting was daily determined using the trypan blue exclusion with a VicellXR and/or by the quantification of the ATP with the CellTiter-Glo assay. For the 3 tested cells lines, the transfection of jouvence allowed cells to proliferate more rapidly. At 160 hours post-seeding, HCT116 h-jou transfected cells reached nearly 5 million cells vs only 2 million cells for the HCT116 empty plasmid cells: more than two folds (counted via the ViCellXR) (Figure 2A). The luminescence assay led to a similar difference (Figure 2B), although it is less pronounced. Similar result was observed for the HEK293-jouvence stably transfected cells compared to control cells (Figure 2C-2D) with an increase of about $50 \%$ of the cell number. Similarly, the Caco2 h-jou stably transfected cells were also more numerous than the corresponding empty plasmid cells (an increase of about $350 \%$ ) (Figure 2E). Therefore, we 
conclude that the overexpression of the $h$-jou importantly stimulates the proliferation of the stably transfected cells. In parallel, to support these results, the corresponding level of $h$-jou was assessed by RT-qPCR. HCT116 transfected cells were overexpressing jou for more than 300 fold compared to the vector (empty-plasmid) transfected cells (Figure 2F). Similarly, Caco2 h-jou transfected cells were enriched with jou for nearly 200 fold (Figure 2G), while the HEK293 $h$-jou transfected cells had a fold-change of more than 1000 compared to the control cells (Figure 2H).

\section{Overexpression of jouvence by Lentivirus also increases the proliferation of the cell.}

As demonstrated above, the overexpression of jouvence leads to an increase of the proliferation of the cells. However, up to now, this effect has been observed only on stably transfected cell lines through the use of a plasmid and lipofectamine transfection. However, the constraints of this approach do not easily allow the overexpression of the $h$-jou in primary cells. In the aim to study the effect of the overexpression of $h$-jou directly on primary cells, we use the lentivirus approach. After building the lentivirus construct/vector and production of lentiviral particles (see Methods), we transduce the jou-lentivirus on various cells. First, we validate the lentivirus approach in HCT116 and CaCo-2 cells to compare the effect on proliferation to the previous observed data obtained in these cell lines. Using two different MOI (1 and 10), we observed, in HCT116 cells, an increase of cell proliferation determined by two independent readouts (Figure 3A-B), as previously described with the plasmid overexpression. In complement, we also measure by RT-qPCR the level of the $h$-jou. Interestingly we found roughly a 10-fold increase in the amount of jouvence (Figure 3C), with a slight increase according to the MOI concentration. In a step further, we also perform such transduction into the CaCo-2 cell line, and similarly, we observe an increase of their proliferation (Figure 3D-E). These similar results obtained on two independent cell lines validate the lentivirus approach, and consequently allow further exploring such approach on non-cancerous cell line and on primary cells. Then, transduction of jou-lentivirus on immortalized but non-cancerous RPE1 cell line (Human retinal pigmented epithelial cells) leads to a similar increase of cell proliferation (Figure 3F-G). Ultimately, the transduction the jou-lentivirus into primary cells (HUVEC) (Human Umbilical Vein Embryonic Cells) also leads to an increase of proliferation, as evaluated by the quantification of ATP using a luminescence assay (Figure 3H). In summary, the overexpression of $h$-jou using jou-lentivirus transduction system/vector yields to similar stimulation of the proliferation of non-cancerous primary cells. 


\section{Decreasing jouvence level by siRNA reduces the proliferation of the cells}

To assess if the decrease of the amount of $h$-jou could lead to a modified phenotype, the knockdown of $h$-jou was performed by transiently transfecting a $h$-jou specific siRNA to the HCT116 adenocarcinoma cell line. Briefly, a double siRNA transfection was performed: the first reverse transfection was made on HCT116 at the time of plating the cells into the culture plates, followed by a siRNA second transfection after 48 hours of culture. The number of cells was assessed after 72 or 96 hours depending of the tested cell lines. The negative siRNA mismatch control was also performed on the same conditions. The HCT116 transfected cells with jou-siRNA were proliferating in a lower rate compared to their two respective controls: the non-transfected cells, but treated with the lipofectamine/RNAiMax only (Co), or treated with the negative Si-RNA control ( $\mathrm{Si}-\mathrm{Co}$ ), with the higher difference at 72 hours posttransfection (Figure 4A). The decrease of the amount of specific snoRNA by the siRNA was confirmed by RT-qPCR with a nearly $40 \%$ decrease of the endogenous $h$-jou at 72 hours posttransfection (Figure 4B). Therefore, we conclude that the partial inhibition (a decrease of 40\%) of the $h$-jou was sufficient to decrease the proliferation of the cells. To assess if this cellular effect of the decrease of the $h$-jou is not cell type specific, we perform similar experiments on other well-established cell lines. Similarly, striking decreases of the cell number were observed for the MCF7 cells (a breast cancer cell line) (Figures 4C-D), the U87-MG cells (a glioblastoma cell line) (Figures 4E-F), and the A549 lung cancer cell line when the $h$-jou is knocked-down (Figures 4G-H). Finally, since the four cell types investigated above are all immortalized cancerous cells, we wondered if similar effects can be observed in primary non-cancerous cells. The $h$-jou knock-down on HUVEC induces a similar decrease of cell proliferation determined both by cell counting ViCellXR (Figure 4I), and by ATP measurement (CellTiter-Glo) (Figure $4 \mathrm{~J})$. These similar results obtained in several different cancerous cell lines as well as on primary cells indicate that a normal and physiological level of $h$-jou is required for the proper cell proliferation. Indeed, increasing the level of $h$-jou stimulates the proliferation, while inversely decreasing its level by siRNA inhibits the proliferation of the cells. It also indicates that $h$-jou is functional in all these cell lines derived from different organs and tissues, although $h$-jou is only expressed at a low level (see Figure 1C).

\section{HCT116 overexpressing $\boldsymbol{h}$-jou presents a genomic signature of dedifferentiation.}

In order to initiate the elucidation of the genetic and molecular mechanisms of the snoRNA-jouvence that could be responsible for these phenotypes, we have characterized the cells on a whole transcriptomic level. A RNA-seq analysis was performed on HCT116 cells, 
comparing the $h$-jou stably transfected cells to their empty plasmid control. The comparison reveals a set of 5918 Differentially Expressed Genes (DEG), with 2974 up-regulated genes and 2944 down-regulated genes (Figure 5A). Based on the fixed p-value, Table 1A shows the list of 19 most up-regulated genes (for the complete list of the up-regulated genes, see the Suppl. Table S1), while the Table 1B shows the list of 19 most down-regulated genes (see Suppl. Table S2 for the complete list of down-regulated genes). The cluster analysis (Heat-map) of differentially expressed genes briefly resumes the up- and down-regulated genes (Figure 5B). To get more precise information about these multiple deregulated genes, the statistical enrichment of DEG in KEGG pathway $[14,15]$ shows an important enrichment in metabolic pathways, with more than 400 DEG with a relatively low Rich Factor but with a high q-value (adjusted p-value) (Figure 5C). Other pathways were also enriched (with high q values), such as RAS signaling pathway, PI3K-AKT pathway, regulation of actin cytoskeleton pathway, pathways in cancer, and the ribosome pathway having the higher degree of enrichment, with a Rich Factor of more than 0,7. Again here, based on the p-value, Table 1C shows the list of the 18 most affected KEGG pathways (see Suppl. Table S3 for the complete list of affected KEGG pathways).

More specifically, among the 2974 up-regulated genes, an important number of them harboring the best adjusted p-values are found to be correlated with a dedifferentiation of the cells [16]. Notably, these genes are up-regulated, as in an EMT (Epithelial-Mesenchymal Transition) context, which is controlled by four major interconnected regulatory networks [1719]. For examples, among them, the VGF nerve growth factor inducible (VGF) known to play a role in the cell plasticity and to induce the transcription factor TWIST1, which facilitate EMT in cancer cells [20]. The Kit Ligand (KITLG), a ligand for the receptor-type protein-tyrosine kinase plays an essential role in cell survival, proliferation, hematopoiesis and stem cell maintenance [21]. The nuclear receptor subfamily 2, group F, member 1 (NR2F1), is a nuclear hormone receptor and a transcriptional regulator. It is associated with stem cells, and acquisition of stem-like properties and quiescence [22]. Transgelin 2 (TAGLN2), an actin-binding protein, is significantly induced in hypoxic cancer cells, while Snail1 is simultaneously increased, and thus, inducing the EMT by downregulating E-cadherin [21,23]. The solute carrier family 1 (glial high affinity glutamate transporter), member 3 (SLC1A3), a glutamate transporter mediates the inter-niche stem cell activation [24]. The early growth response 1 (EGR1) factor has a role in controlling cell plasticity, which has been involved in TGF $\beta$ 1-induced EMT [25]. The Kruppel-like factor 2 (KLF2) family are considered as key transcription factors implicated in 
self-renewal of embryonic stem cells [26]. All of these key genes, among others, are considered as landmark of EMT.

Inversely, 2944 genes are under-expressed. Interestingly, also here, an important number of these down-regulated genes have been shown to be deregulated during the EMT, which again suggests that the overexpression of $h$-jou induces EMT. For examples, the Tubulointerstitial nephritis antigen-like 1 (TINAGL1) decreases the secretion of metastasissuppressive proteins including insulin-like growth factor binding protein 4 (IGFBP4) [27]. The hepatocyte nuclear factor $4 \alpha$ (HNF4A) is under-expressed (fold-change 0,02), while the EMT in hepatocytes correlates with the down-regulation of the hepatic differentiation key factors HNFs [28]. The Keratin 13 (KRT13) is epigenetically suppressed during Transforming Growth Factor- $\beta$ 1-induced epithelial-mesenchymal transition [29]. The catenin (cadherin-associated protein), alpha-like 1 (CTNNAL1), an epithelial marker is also under-expressed [30]. The Myosin binding protein $\mathrm{H}(\mathrm{MYBPH})$ which inhibits cell motility and metastasis, and then linked to an EMT phenotype, is also under-expressed [31]. In addition Claudin 9 (CLDN9), an epithelial marker [32] is under-regulated in the $h$-jou overexpressing cells, while it is found to be overexpressed in the siRNA transfected HCT116 cells (see below). Annexin 8 (ANXA8) is down-regulated (with a fold-change of 0,1 ). This last has been shown to be transcriptionally down-regulated by epidermal growth factor (EGF), which correlates with the morphologic changes of the epithelial-to-mesenchymal transition (EMT) [33], along with tumor dedifferentiation. To finish the selected list of markers, the epithelial marker Protocadherin 1 (PCDH1) is also down-regulated. PCDH1 binds to SMAD3 and suppresses TGF $\beta 1$-induced gene transcription [34].

In addition, the overexpressing $h$-jou transfected cells are also enriched in CSC (Cancer Stem Cells) potential markers, such as : SOX4, SOX8, CD44, MSI-2, EpCAM to name but of few (see Figure 7). Sox family expression has been correlated with mesenchymal traits and loss of epithelial features. SOX4 plays a role in TGF $\beta$-induced EMT and confers stem cell characteristics [35]. SOX8 regulates CSC properties and EMT via Wnt $\beta$-catenin pathway [36]. The cell surface antigen CD44, Musashi RNA-binding protein 2 (MSI-2) and the Epithelial Cell Adhesion molecule (EpCAM) are also potential CSC markers [37], and are found to be overexpressed in $h$-jou transfected HCT116 cells. In addition, an important number of KEGG pathways are significantly deregulated (Figure 5C and Suppl. Table S3), as the Insulin Secretion Pathway, and the Insulin signaling pathway. Many of the lipid metabolic pathways are also deregulated, such as Glycerophospholipid pathway, Glycerolipid metabolism, sphingolipid 
metabolism pathway. The Longevity regulating pathways are also affected, with genes like IRS2, AKT3, IGF1R, NFkB1, FOXO1, and RPTOR. These pathways are linked (directly or indirectly) to the EMT, the plasticity and the longevity of the cells. Finally, to support the RNASeq analysis, we also validated, by RT-qPCR some of the DEG (Suppl. Figure 1A).

\section{Knockdown of $h$-jou by siRNA decreases the ribosome biogenesis and spliceosome}

Similarly, a RNA-seq analysis was performed on HCT116 cells with a depletion of jouvence. The comparison of the HCT116 h-jou knocked-down by siRNA transfected cells compared to the HCT116 non-transfected cells (treated with lipofectamine/RNAiMax only) reveals a set of 6263 Differentially Expressed Genes (DEG), with 3098 up-regulated genes and 3165 down-regulated genes (Figure 6A). The Table 2A shows the list of 19 most up-regulated genes (for the complete list of the up-regulated genes, see the Suppl. Table S4). The cluster analysis of differentially expressed genes briefly resumes the up- and down-regulated genes (Figure 6B). KEGG analysis pathways enrichment presents an enrichment in the Metabolic pathways, with the highest number of DEG ( 400) (Figure 6C). However, the Spliceosome and Ribosome KEGG pathways had the highest Rich Factor (around 0,7). Pathways like RNA transport, Ubiquitin Mediated proteolysis, Protein processing in the endoplasmic reticulum, Pathways in cancer, MAPK signaling pathway, Hippo pathway, to name but a few, were also enriched with low q values (around 0) and a Rich Factors varying from 0,4 to nearly 0,6.

More specifically, the Solute Carrier family 7 (anionic amino acid transporter light chain, xc-system), member 11 (SLC7A11) and interleukin 20 receptor- $\beta$ (IL20RB) are upregulated in the siRNA condition. Kelch domain containing 7B (KLHDC7B), which has been associated with gene modulation activity in the Interferon signaling pathway [38], is also upregulated after $h$-jou knock-down (fold-change of 83,3). Roundabout, axon guidance receptor, homolog 4 Drosophila (ROBO4) which increases cell adhesion [39] and correlates with an epithelial phenotype, is upregulated (with a fold-change of 73,09). Moreover, Cadherin 15, type 1, M-cadherin (CDH15) and Claudin 2 (CLDN2), which are epithelial markers, are overexpressed when $h$-jou is depleted. This suggests that the inhibition of the snoRNA could lead to more pronounced epithelial phenotype (MET) while inversely, its overexpression favors the EMT.

On the other hand, the knockdown of $h$-jou leads to the down-regulation of 3165 , with a majority of down-regulated genes belonging the ribosome or spliceosome pathways. Table 2B shows the list of 19 most down-regulated genes (for the complete list of the down-regulated genes, see the Suppl. Table S5). This refers to the implication of the H/ACA box snoRNAs in 
the ribosome biogenesis, the modification and processing of ribosomal RNA precursors [1-4]. More specifically, splicing factors are down-regulated such as serine/arginine-rich splicing factor 4 (SRSF4), serine/arginine-rich splicing factor 11 (SRSF11), splicing factor 3b, subunit 1, 155kDa (SF3B1) and splicing factor 3b, subunit 2, 145kDa (SF3B2) (Suppl. Table S5). Some ribonucleoproteins are also down-regulated: heterogeneous nuclear ribonucleoprotein $\mathrm{H} 1$ (HNRNPH1), small nuclear ribonucleoprotein 70kDa (U1) (SNRNP70), small nuclear ribonucleoprotein polypeptide E (SNRPE) (Table 2B and Suppl. Table S5). In other words, the main deregulated KEGG pathways (with the highest Rich Factors) are the Ribosome and the Spliceosome pathways (Table 2C). More strikingly, the depletion of $h$-jou leads to the downderegulation of the majority of the genes of these pathways. For the Ribosome pathway, 90 genes were down-regulated over the 99 genes. For the Spliceosome pathway, while the total number of genes is 90 , among them, 80 were down-regulated. These effects perfectly correlate with the main known function of the snoRNAs in guiding chemical modifications of other RNAs, mainly rRNAs, transfer RNAs and small nuclear RNAs, through their function within their related RNPs. Finally, we have also validated, by RT-qPCR, some of these DEG: CLN2, HMGCR, PRPF3, SRSF4, DHRS2, IL20RB, SLC7A11 (Suppl. Figure 1B).

\section{Several genes are deregulated in opposite direction when comparing overexpression and depletion of jou.}

The RNA-Seq analysis has revealed that several genes are differentially expressed (DEG) either up or down, and so, both in overexpression or in knockdown of $h$-jou, related to a significant number of enriched KEGG pathways. Then, we wonder if the genes that are upregulated in $h$-jou overexpression condition are inversely downregulated in the inverse jouvence-knocked-down condition, and vice-versa. Thus, when the upregulated genes in the jouvence-overexpression condition were compared to the down-regulated genes in the jouvence depletion (siRNA condition), we found 1102 DEG in common (see Suppl. Table S7 for the list of genes). Conversely, the comparison of the down-regulated genes in jouvence overexpression condition versus the overexpressed genes in jouvence depletion (siRNA condition) led to a number of 868 DEG in common (see Suppl. Table S8 for the list of genes). For examples, among the 3098 overexpressed genes, the genes DHRS2, SLC7A11, IL20RB, KLHDC7B, ROBO4, CDH15, CDLN2, that are up-regulated when the $h$-jou is inhibited were found to be down-regulated in the inverse condition of $h$-jou stably transfected cells (overexpression). Briefly, Dehydrogenase/reductase (SDR family) member 2 (DHRS2) which inhibits cell growth and motility [40], was overexpressed in the siRNA cells, which correlates with the observed 
phenotype after siRNA transfection, where proliferation rate decreased significantly at 72 hours post-transfection-1. Interestingly, these results indicate that $h$-jou leads to the up- or downregulation of a similar set of genes depending of its over- or under-expression. Consequently, it suggests that these multiple genes are directly sensitive to the level of $h$-jou, and therefore are likely not only a consequence of the deregulation of other genes.

\section{Discussion}

We have characterized a new snoRNA named jouvence in human, which was not yet annotated in the genome. jouvence is localized on the chromosome 11 in a large intron of the gene TEAD1. As previously reported [13], in addition to some tissues, $h$-jou is expressed, although weakly, in all tested various well-established cell lines. To gain insights about the cellular role of jouvence, we first overexpress $h$-jou into different cancerous and non-cancerous cell lines. Interestingly, the overexpression of jouvence leads to an important increase of the proliferation of the cells, yielding to about twice more cells within about one week. More importantly, this effect is observed with two independent approaches to overexpress $h$-jou, first through a stably transfected-plasmid and second through a transduction with a lentivirus vector. This second result obtained with an integrative lentivirus indicates that a snoRNA can be successfully carried by lentivirus. Inversely, the decrease (knockdown) of $h$-jou by transiently transfected specific siRNA, leads to an opposite phenotype characterized by a rapid decrease of cell proliferation.

To investigate further the role of the $h$-jou and more particularly its molecular mechanism, we perform a transcriptomic analysis (RNA-Seq). The overexpression of the $h$-jou leads to the up- and down-regulation of many genes. Among them, several deregulated genes, as Twist, SNAIL/SMUG/SMUC, ZEB, Fibonectin, suggest a dedifferentiation signature of the cell (Figure 7). This cellular effect could be comparable, at least in part, to a rejuvenation of the cells [41]. It could also remind, in some ways and in a larger context, the organismal phenotype observed in Drosophila, an extension of lifespan [13]. On the other hand, the RNA-Seq performed on the decrease of $h$-jou through the knockdown by siRNA, also shown that several genes are deregulated, both up and down, while the bioinformatics analysis has revealed that the main affected KEGG pathways are, in addition to the metabolic pathway, the ribosomes biogenesis and the spliceosome. These results are in agreement with the canonical role of a H/ACA box snoRNA, known to be involved in the regulation of the ribosome biogenesis and the modification of the ribosomal RNA [1-4]. More precisely, according to the KEGG 
pathways, 99/138 genes of the ribosome pathway are affected, and among them, 91/99 are downregulated. For the spliceosome pathway, 90/134 genes are affected, and among them, 80/90 are downregulated. In overview, these results indicate a clear and an almost complete breakdown of the ribosome and the spliceosome pathways, which consequently might explain the decrease of cell proliferation. It has been reported that repressing or perturbing the ribosome biogenesis decreases the growth of the cells [42], and/or even could drive cells into senescence [43].

The Epithelial to Mesenchymal Transition (EMT) is a process by which epithelial cells lose cell-cell adhesion, and gain migratory and invasive properties to become mesenchymal stem cells $[18,19,21]$. The cancer cell lines with mesenchymal phenotype are characterized by under-expression of different genes such as claudins, cadherins, occludins, among others. In addition, genes such as Fibronectin, Jagged 1 are overexpressed, along with the three main EMT transcriptional factors : SNAIL/SLUG/SMUC, Twist and ZEB [17]. These factors can act together to induce EMT. Indeed, human SNAIL1 (SNAI1) protein encoded by SNAI1/SNA gene represses the transcription of E-cadherin/CDH1 gene (Figure 7). Human SNAIL2 (SNAI2) protein encoded by SNAI2/SLUG gene induces the first phase of epithelialmesenchymal transition (EMT), including desmosome dissociation, cell spreading, and initiation of cell separation [17]. Twist 1 activates other EMT-inducing transcription factors to suppress E-cadherin and promote EMT and tumor metastasis. Twist 1 needs to induce Snail2 to suppress the epithelial branch of the EMT program, while Twist1 and Snail2 act together to promote EMT [17]. ZEB is regulated by multiple signaling pathways, such as WNT, NF- $\mathrm{B}$, TGF- $\beta$, COX2, HIF, among others [17,44].

Remarkably, the overexpression of the new H/ACA $h$-jou seems to be sufficient by itself to re-orientate the cells toward the dedifferentiation, although the HCT116 cells (an adenocarcinoma line from the gut) is originally cancerous cells. Nevertheless, the overexpression of $h$-jou seems to superimpose a signature of dedifferentiation. Indeed, several key genes involved in the EMT (Epithelial-Mesenchymal Transition), as TWIST, SNAIL/SMUG/SMUC, ZEB, Jagged and fibronectin, are upregulated, while some of their target genes such as claudin, E-cadherin, annexin, and $\alpha$-catenin are downregulated (Figure 7) $[19,21,45]$. Nonetheless and alternatively, at this stage of this study, we could not exclude that this EMT process could rather be interpreted as a step toward the acquisition of a cancer stem cell (CSC) phenotype. Indeed, some evidence suggests that snoRNAs may also play a role in cancer stem cells (CSC) [12]. For example, ALDH1 has been demonstrated to be a CSC marker [46]. Here, several deregulated genes could also suggest a CSC mechanism, as for instance, few 
ALDH1-subunits, as ALDH1A3, ALDH1B1, and ALDH1L2. Other genes as SOX4, SOX8, CD44, MSI-2 and EPCAM, which are also deregulated in $h$-jou depletion have also been considered as potential CSC markers [47] (Figure 7). Further experiments will be required to discern between a CSC trend rather than an EMT dedifferentiation trend.

Based on its primary sequence, jouvence is a snoRNA of type H/ACA box, generally known to perform pseudouridylation [1]. Pseudouridylation is a post-transcriptional isomerization reaction that converts uridine to pseudouridine ( $\Psi)$ [48]. This last is present in several different types of RNAs, including coding and noncoding RNAs [5,49]. $\Psi$ is particularly concentrated in rRNA, in which it plays an important role in protein translation, as well as in spliceosome snRNAs, in which it is involved in pre-mRNA splicing. Here, disrupting the level of $h$-jou, either by overexpression or by decreasing its expression, leads to the deregulation of a quite huge number of genes (about 6000: about half up and half down), as revealed by the RNA-seq analysis. Interestingly, among them, about 1000 genes (868 and 1102 respectively) are deregulated in the opposite direction when we compare $h$-jou overexpression with its depletion by siRNA (or vice-versa), suggesting that the expression level of these genes follows directly the level of jou, and consequently might be likely directly regulated by the snoRNAjouvence. In counterpart, it suggests that the remaining deregulated genes $(\sim 5000)$ are rather likely a consequence of the deregulation of these "primary" 1000 genes, or in another term, a secondary effect. Further experiments will be required to decipher the precise molecular mechanisms of jouvence which leads to the deregulation of these multiple genes, either due to transcription or translation modifications, chromatin remodelling, RNA stability, or else.

In addition to the well-known canonical function of the snoRNA in various RNA modifications, more recently, some snoRNAs have been involved in stress response and metabolic homeostasis. For examples, the snoRNA U32a, U33 and U35a are mediators of oxidative stress [50], while the same snoRNAs seem also to regulate the systemic glucose metabolism [51]. In the same way, Brandis et al. [52] has shown that the box C/D snoRNA U60 regulates intracellular cholesterol trafficking between the plasma membrane and the endoplasmic reticulum. Similarly, the snoRNA U17 regulates the cellular cholesterol trafficking through the hypoxia-upregulated mitochondrial regulator (HUMMR) by acting on its target mRNA [53]. Here, the modification of the level of the $h$-jou, either its overexpression or its depletion leads to a strong deregulation of metabolic pathways as revealed by the KEGG analysis (about 400 genes). Interestingly, among them, the 3-hydroxy-3-methylglutaryl-CoA reductase (HMGCR), one of the key limiting enzyme involved in cholesterol synthesis (which is also the target of the statin, an anti-cholesterol), is upregulated when jouvence is 
overexpressed. Inversely, it is downregulated when jouvence is depleted, suggesting that this crucial gene, involved in the regulation of the cholesterol level, is likely directly regulated by jouvence.

Several snoRNAs (both C/D and H/ACA boxes) have also been involved in many biological cancer processes, as tumor initiation, invasion, metastasis, and/or proliferative signalling [10,11]. For examples, some snoRNAs have been associated with the p53 pathway, a well-known tumor suppressor [54]. Here on HCT116 cells, the p53 gene itself is not directly deregulated, but several of its regulators are affected. Another crucial gene involved in cancer and more particularly in ribosome biogenesis is myc [55]. Indeed, in breast cancer, the Myc oncogene upregulates the expression level of fibrillarin, an enzymatic small nucleolar ribonucleoprotein (snoRNP), which led to elevated snoRNAs biogenesis, and consequently inducing p53 suppression [56]. Interestingly, in various model organism, as C. elegans [57], Drosophila [58], and mouse [59], a link between Myc, the ribosomes biogenesis, and lifespan has been established. Furthermore, in Drosophila, it has been proposed that snoRNAs are a novel class of biologically relevant Myc targets [60]. Here, in HCT116 cells overexpressing jouvence, MYC and MYCL are upregulated as well as some of its multiple regulators/interactors. Moreover, the depletion of some snoRNAs, as U3 and U8 led to ribosomes dysfunction [61]. Interestingly, the ribosome biogenesis is strongly affected in jouvence depleted HCT116 cells. Finally, the SNORD76, a box C/D snoRNA has been shown to act as a tumor suppressor in glioblastoma [62], a similar effect observed with the depletion of jouvence in U87-MG glioblastoma cell line. In conclusion, as previously suggested [10,11], snoRNAs have the potential to become cancer biomarkers, and even may potentially become major cancer therapeutic targets in the near future. In such way, the snoRNA-jouvence is surely another good and promising candidate.

\section{Methods}

\section{Cell lines and culture conditions}

Cancer cell lines were obtained from the American Type Culture Collection (Rockville, MD, USA) and were cultured according to the supplier's instructions. Primary Human Umbilical Vein Endothelial Cells (HUVEC) isolated from the vein of the umbilical cord were obtained from Promocell (Germany). HCT116 cells were cultured in McCoy's 5A medium supplemented with 10\% Fetal Bovine Serum and 1\% glutamine. hTERT-RPE1 and Caco 2 cells were maintained in Dulbecco's modified Eagle's medium: nutrient mixture F-12 supplemented 
with 10\% FBS and 1\% glutamine. HEK293 and U87-MG cells were cultured in DMEM 1X medium supplemented with $10 \%$ of FBS and 1\% glutamine. MCF7 and A549 cells were cultured in RPMI 1640 supplemented with 10\% Fetal Bovine Serum and 1\% glutamine. HUVECs were grown in Endothelial Cell Growth Medium 2 which is low-serum (2\% V/V) media optimized for the cultivation of endothelial cells from large blood vessels. Cells were incubated at $37^{\circ} \mathrm{C}$ in a humidified atmosphere containing $5 \% \mathrm{CO} 2$.

\section{Stable cell transfections}

In order to generate stable cell lines that overexpress the human snoRNA-jouvence, the $h$-jou was cloned in the pcDNA3.1 Zeo + plasmid (ThermoFisher Scientific, USA) between EcoRI sites, in 5' - 3' direction behind a T7 promoter. HCT116, Caco2 or HEK 293 cells were transfected with the empty plasmid, serving as a control or with the plasmid containing $h$-jou cloned sequence. Briefly, cells were plated in 12-well plates and allowed to grow 24 hours and reach nearly 60 to $70 \%$ of confluence at the time of transfection. Cells were transfected with the corresponding plasmid, using the Lipofectamine 3000 (ThermoFisher Scientific, USA) at a ratio of 1:2 for the DNA:lipofectamine. Lipofectamine 3000, p3000 and the plasmids were previously diluted in OptiMEM 1X (Gibco) (a Reduced-Serum Medium). The medium was changed 24 hours after the transfection, and cells were allowed to grow for another additional 24 hours before being split to a lower concentration. 72 hours post-transfection, cells were treated with Zeocine (ThermoFisher Scientific) $(75 \mu \mathrm{g} / \mathrm{mL}, 200 \mu \mathrm{g} / \mathrm{mL}$, and $150 \mu \mathrm{g} / \mathrm{mL}$ for the HCT116, Caco2 and HEK 293 cells respectively). The selection of stably transfected cells was conducted over a period of nearly 4 to 5 weeks with the addition of Zeocine every 3 to 4 days. Stable clones expressing either the empty plasmid or the plasmid with the human snoRNA were selected, verified by RT-qPCR (TaqMan) before their use in the different experiments.

\section{Lentivirus preparation and in-vitro infection (transduction)}

Classical Lentiviral integrative vector expressing the snoRNA-jouvence placed downstream to the U6 promoter (pLV.U6.hsnoRNA-jouvence), and containing the puromycine selection marker was generated by Flash Therapeutics/Vectalys (Toulouse, France). Cell infections were carried out according to Flash Therapeutics/Vectalys recommendations. For the HCT116 cell line, two MOI have been tested (MOI-1 and MOI-10). Since MOI-10 gives good results and the highest snoRNA-jou expression level, we uses MOI-10 for all other cell lines. For all cell lines, a puromycine selection has been performed for 48 hours. In such condition, all non- 
transduced cells were eliminated (performed in parallel on non-lentivirus transduced cells, as control).

\section{Transfection of siRNA}

The effect of the knockdown of the snoRNA-jouvence on the HCT116 cells was assessed by the transfection of short interfering RNA (siRNA). First, two different silencer selected siRNAs (Lock-Nucleotid-Acid siRNA or LNA-siRNA, shortly named siRNA) were tested (siRNA-1, antisense sequence: 3'-UCCUCUGUCCACAAUAGCC-5', Cat nb : 4399665), and (siRNA-2, antisense sequence: 3'-UCAAGACCAAUCACCAUGU-5', Cat nb : 4399665), as well as a non-targeting siRNA-control used for the specificity of the knockdown (Cat nb: 4390843) (ThermoFisher Scientific, USA). These two siRNAs (as well as the siRNA-control) were independently transfected into the cells in a 12-wells format. Cell suspensions of $0,25 \times 10^{6}$ HCT116 cells per well were directly transfected with the corresponding siRNA (reverse transfection) at a final concentration of $10 \mathrm{nM}$ per well, using the Lipofectamine RNAiMAX transfection reagent (ThermoFisher Scientific, USA). 48 hours after the first siRNA reverse transfection, a forward transfection was performed on the adherent cells (at the same concentration as the first reverse transfection). After $24 \mathrm{~h}$, the medium was changed and cells were kept in complete medium. Then, the cells were counted at different time points (days postinfection), depending of the cells lines and compared to the different controls. Cell pellets (for the RNA extraction and RNA-seq analysis) were made 48 hours post-transfection-2 (96h after the first reverse transfection). Conditions were performed in triplicates and the specific knockdown of the snoRNA-jouvence was validated by standard RT-qPCR (TaqMan).

\section{VicellXR counting}

The proliferation rate of the stably transfected cells (empty plasmid cells and snoRNA overexpressing cells) was analysed by cell counting. Cells were seeded in 6-well plates (in triplicates), with the following number of cells per well: $0,475 \times 10^{6}$ HCTT16 cells per well ; $0,5 \times 10^{6}$ Caco2 cells per well and 0,2 X $10^{6}$ cells per well for the HEK 293 cells. The experiment was conducted over a period of 96 to 160h. Briefly, the supernatant was harvested and cells washed with PBS 1X. Then, cells were trypsinized with $300 \mu 1$ of $0.05 \%$ trypsin/EDTA (Gibco) per well. Trypsine was inactivated with $700 \mu \mathrm{L}$ of the corresponding medium per well, and were cells then counted with the VicellXR using trypan blue to determine cell viability (Cell viability Analyzer, Beckman Coulter). 


\section{CellTiter-Glo}

A luminescent cell viability assay (CellTiter-Glo, Promega, USA) was assessed to determine the cell proliferation rate based on quantitation of the ATP, an indicator of metabolically active cells. Three thousand cells were plated in each well of a 96-well white plate with clear bottom (Costar 3610, Corning Incorporated, USA). Each cell condition was plated in 6 or 10 wells. Cells were allowed to adhere for $24 \mathrm{~h}$, before counting. The homogeneous assay procedure involves addition of $100 \mu \mathrm{L}$ of the CellTiter-Glo reagent directly to the cells cultured in $100 \mu \mathrm{L}$ of their corresponding complete medium, before measuring the relative luminescence using a multiplate reader (POLAR Star Omega BMG LABTECH). The experiment was conducted over a week, with counting performed every 24h for HCT116 and HEK293, and at 96 hours for the lentivirus transduction.

\section{RNA extraction and Reverse Transcription (RT)}

Total RNA from human cell lines were extracted using NucleoSpin RNA Plus columns (Macherey-Nagel, France), according to the manufacturer's instructions, as in Soulé et al., 2020 [13]. Extracted RNAs were verified for the absence of genomic DNA contamination, by performing a RT-PCR using the ribosomal gene RP49. Contaminated samples were therefore treated with RQ1 DNase (Promega, USA) and cleaned with the NucleoSpin RNA Clean-UP (Macherey-Nagel). Two micrograms of total RNA were used for the synthesis of cDNA with oligo-dT (used for Sybr Green RT-qPCR) or random primers (used for TaqMan RT-qPCR for the detection of the snoRNA expression). The M-MULV reverse transcriptase (Promega, USA) was used and the RT was performed in a final volume of $25 \mu \mathrm{L}$. The thermal cycling conditions were $37^{\circ} \mathrm{C}$ for $50 \mathrm{~min}$ followed by $15 \mathrm{~min}$ at $70^{\circ} \mathrm{C}$. RNase $\mathrm{H}$ (Invitrogen, USA) was performed to digest RNA-DNA hybrids.

\section{RT-qPCR (Real Time Quantitative Polymerase Chain Reaction) on selected genes}

The differential expression of the snoRNA and selected genes was analysed by real-time PCR (QuantStudio 3, Applied Biosystems, France). The expression of the snoRNA was detected with TaqMan customized probes (Applied Biosciences, Life Technologies) and the TaqMan Universal Master Mix II, no UNG (Applied Biosystems). All conditions were normalized to the GAPDH control gene. The Sybr Green RT-qPCR was performed for all the other genes with the Power UP SYBR-Green PCR Master Mix, according to the manufacturer's instructions (Applied Biosystems). Primers were designed using the "Primer 3 Plus" software (the primer sequences will be provided upon request). All conditions were normalized to the RPLP0 control 
gene. The results were analysed using the $2-\Delta \Delta \mathrm{Ct}$ method, and displayed as the fold change compared to the control gene.

\section{Transcriptomic analysis (RNA-seq)}

The Transcriptomic analysis (RNA-seq) was performed by Novogene (China). Briefly, according to Novogene, total RNA was extracted and after sample quality control, libraries were generated and checked for quality. Then the libraries were sequenced on an Illumina Hiseq platform and $125 \mathrm{bp} / 150 \mathrm{bp}$ paired-end reads were generated. The resulting data were controlled, and analyzed with bioinformatic tools. The reference genome and gene model annotation files were downloaded from genome website directly. Index of the reference genome was built using Bowtie v2.2.3 and paired-end clean reads were aligned to the reference genome using TopHat v2.0.12. Novogene uses TopHat as the mapping tool because TopHat can generate a database of splice junctions based on the gene model annotation file and thus a better mapping result than other non-splice mapping tools. For the quantification of gene expression level, Novogene uses HTSeq v0.6.1 to count the reads numbers mapped to each gene. Then FPKM of each gene was calculated based on the length of the gene and reads count mapped to this gene. FPKM, expected number of Fragments Per Kilobase of transcript sequence per Millions base pairs sequenced, considers the effect of sequencing depth and gene length for the reads count at the same time, and is currently the most commonly used method for estimating gene expression levels. Novogene uses DESeq R package (1.18.0) to perform differential expression analysis of two conditions (three biological replicates per condition). DESeq provide statistical routines for determining differential expression in digital gene expression data using a model based on the negative binomial distribution. Prior to differential gene expression analysis, for each sequenced library, the read counts were adjusted by edgeR program package through one scaling normalized factor. The $\mathrm{P}$ values were adjusted using the Benjamini \& Hochberg method. Corrected P-value of 0,05 and $\log 2$ (Fold-change) of 1 were set as the threshold for significantly differential expression. The biological variation was eliminated (case with biological replicates), and the threshold was therefore normally set as p adjusted $<0,05$. Gene Ontology (GO) enrichment analysis of differentially expressed genes was implemented by the GOseq R package, in which gene length bias was corrected. GO terms with corrected pvalue less than 0,05 were considered significantly enriched by differential expressed genes. For the KEGG database $[14,15]$, Novogene uses KOBAS software to test the statistical enrichment of differential expression genes in KEGG pathways. We acknowledge Novogene (China) for the assistance in the Method descriptions of the transcriptomic analysis. 
Statistical Analysis: All data were analysed statistically using one-tailed unpaired t-test, with GraphPad Prism ${ }^{\mathrm{TM}}$ software.

\section{Acknowledgements}

We thank K. Sidelarbi and L. Mellottée for their technical assistance. This work was supported by Ninovax (a subsidiary of Truffle Capital, Paris), and by the CNRS to J. Bignon and JR Martin.

Author contributions: JRM and JB conceived and designed the experiments. FEK and JB performed experiments. FEK, JB, JRM analysed the data. JRM wrote the manuscript with input from all authors.

Conflict of interest: The authors declare no conflict of interests.

\section{Supplementary Information:}

One Supplementary Figure and eight Supplementary Tables (S1 to S8) containing the RNASeq Data-analysis are available (Excel files).

\section{References}

1. Kiss, T. Small nucleolar RNAs: an abundant group of noncoding RNAs with diverse cellular functions. Cell, 109, 145-148 (2002).

2. Gardner, P. P., Bateman, A. \& Poole, A.M. SnoPatrol: how many snoRNA genes are there? J Biol. 9, 4 (2010).

3. Ye, K. H/ACA guide RNAs, proteins and complexes. Curr Opin Struct Biol. 17, 287-292. (2007). 
4. Kiss, T., Fayet-Lebaron, E. \& Jády, B.E. Box H/ACA small ribonucleoproteins. Mol Cell. 37, 597-606 (2010).

5. McMahon, M., Contreras, A. \& Ruggero, D. Small RNAs with big implications: new insights into H/ACA snoRNA function and their role in human disease. Wiley Interdiscip Rev RNA 6, 173-189 (2015).

6. Mitchell, J. R., Wood, E. \& Collins, K. A telomerase component is defective in the human disease dyskeratosis congenita. Nature 402, 551-555 (1999).

7. Vulliamy, T. et al. The RNA component of telomerase is mutated in autosomal dominant dyskeratosis congenita. Nature 413, 432-435 (2001).

8. Cavaillé, J. et al. Identification of brain-specific and imprinted small nucleolar RNA genes exhibiting an unusual genomic organization. Proc Natl Acad Sci USA 97, 14311-14316 (2000).

9. Kishore, S. \& Stamm, S. The snoRNA HBII-52 regulates alternative splicing of the serotonin receptor 2C. Science 311, 230-232 (2006).

10. Mannoor, K., Liao, J. \& Jiang, F. Small nucleolar RNAs in cancer. Biochim Biophys Acta. 1826, 121-128 (2012).

11. Liang, J., Wen, J., Huang, Z., Chen, X. P., Zhang, B. X. \& Chu, L. Small Nucleolar RNAs: Insight Into Their Function in Cancer. Front Oncol. 9, 587 (2019).

12. Batlle, E. \& Clevers, H. Cancer stem cells revisited. Nat Med. 23, 1124-1134 (2017).

13. Soulé, S., Mellottée, L., Arab, A., Chen, C. \& Martin, J. R. Jouvence a small nucleolar RNA required in the gut extends lifespan in Drosophila. Nat. Commun. 11, 987 (2020).

14. Kanehisa, M., Furumichi, M., Tanabe, M., Sato, Y. \& Morishima, K. KEGG: new perspectives on genomes, pathways, diseases and drugs. Nucleic Acids Res. 45, D353-D361 (2017).

15. Kanehisa, M., Sato, Y., Furumichi, M., Morishima, K. \& Tanabe, M. New approach for understanding genome variations in KEGG. Nucleic Acids Res. 47, D590-D595 (2018).

16. Takahashi, K. \& Yamanaka, S. Induction of pluripotent stem cells from mouse embryonic and adult fibroblast cultures by defined factors. Cell 126, 663-676 (2006).

17. De Craene, B. \& Berx, G. Regulatory networks defining EMT during cancer initiation and progression. Nat Rev Cancer 13, 97-110 (2013).

18. Pastushenko, I. \& Blanpain, C. EMT Transition States during Tumor Progression and Metastasis. Trends Cell Biol. 29, 212-226 (2019).

19. Santos, F., Moreira, C., Nóbrega-Pereira, S. \& Bernardes de Jesus, B. New Insights into the Role of Epithelial-Mesenchymal Transition during Aging. Int J Mol Sci. 20, 891 (2019). 
bioRxiv preprint doi: https://doi.org/10.1101/2020.06.17.157321; this version posted June 18, 2020. The copyright holder for this preprint (which was not certified by peer review) is the author/funder. All rights reserved. No reuse allowed without permission.

20. Hwang, W. et al. Expression of Neuroendocrine Factor VGF in Lung Cancer Cells Confers Resistance to EGFR Kinase Inhibitors and Triggers Epithelial-to-Mesenchymal Transition. Cancer Res. 77, 3013-3026 (2017).

21. Thiery, J. P., Acloque, H., Huang, R. Y. \& Nieto, M. A. Epithelial-mesenchymal transitions in development and disease. Cell 139, 871-890 (2009).

22. Sosa, M. S. et al. NR2F1 controls tumour cell dormancy via SOX9- and RAR $\beta$-driven quiescence programmes. Nat. Commun. 6, 6170 (2015).

23. Kim, I. G., Lee, J. H., Kim, S. Y., Hwang, H. M., Kim, T. R. \& Cho, E. W. Hypoxiainducible transgelin 2 selects epithelial-to-mesenchymal transition and $\gamma$-radiation-resistant subtypes by focal adhesion kinase-associated insulin-like growth factor 1 receptor activation in non-small-cell lung cancer cells. Cancer Sci. 109, 3519-3531 (2018).

24. Reichenbach, B., Classon, J., Aida, T., Tanaka, K., Genander, M. \& Göritz, C. Glutamate transporter Slc1a3 mediates inter-niche stem cell activation during skin growth. EMBO J. 37, pii: e98280 (2018).

25. Shan, L. N., Song, Y. G., Su, D., Liu, Y.L., Shi, X. B. \& Lu, S. J. Early Growth Response Protein-1 Involves in Transforming Growth factor- $\beta 1$ Induced Epithelial-Mesenchymal Transition and Inhibits Migration of Non-Small-Cell Lung Cancer Cells. Asian Pac J Cancer Prev. 16, 4137-4142 (2015).

26. Wang, H., Zhou, Y., Yu, D. \& Zhu, H. Klf2 contributes to the stemness and self-renewal of human bone marrow stromal cells. Cytotechnology. 68, 839-848 (2016).

27. Korpal, M. et al. Direct targeting of Sec23a by miR-200s influences cancer cell secretome and promotes metastatic colonization. Nat Med. 17, 1101-1108 (2011).

28. Sekiya, S. \& Suzuki, A. Direct conversion of mouse fibroblasts to hepatocyte-like cells by defined factors. Nature 475, 390-393 (2011).

29. Hatta, M., Miyake, Y., Uchida, K. \& Yamazaki, J. Keratin 13 gene is epigenetically suppressed during transforming growth factor- $\beta 1$-induced epithelial-mesenchymal transition in a human keratinocyte cell line. Biochem Biophys Res Commun. 496, 381-386 (2018).

30. Tan, M., Liu, C., Huang, W., Deng, L., Qin, X. \& Xiang, Y. CTNNAL1 inhibits ozoneinduced epithelial-mesenchymal transition in human bronchial epithelial cells. Exp Physiol. 103, 1157-1169 (2018).

31. Hosono, Y. et al. MYBPH, a transcriptional target of TTF-1, inhibits ROCK1, and reduces cell motility and metastasis. EMBO J. 31, 481-493 (2012).

32. Gon, Y. et al. NDRG1 is important to maintain the integrity of airway epithelial barrier through claudin-9 expression. Cell Biol Int. 41, 716-725 (2017).

33. Lee, M. J. et al. ANXA8 down-regulation by EGF-FOXO4 signaling is involved in cell scattering and tumor metastasis of cholangiocarcinoma. Gastroenterology 137, 1138-1150. (2009). 
34. Faura Tellez, G. et al. Protocadherin-1 binds to SMAD3 and suppresses TGF- $\beta 1$-induced gene transcription. Am J Physiol Lung Cell Mol Physiol. 309, L725-L735 (2015).

35. Peng, X., Liu, G., Peng, H., Chen, A., Zha, L. \& Wang, Z. SOX4 contributes to TGF- $\beta$ induced epithelial-mesenchymal transition and stem cell characteristics of gastric cancer cells. Genes Dis. 5, 49-61 (2017).

36. Xie, S. L. et al. SOX8 regulates cancer stem-like properties and cisplatin-induced EMT in tongue squamous cell carcinoma by acting on the Wnt/ $\beta$-catenin pathway. Int $J$ Cancer 142, 1252-1265 (2018).

37. Mokrowiecka, A. et al. Expression profiles of cancer stem cell markers: CD133, CD44, Musashi-1 and EpCAM in the cardiac mucosa-Barrett's esophagus-early esophageal adenocarcinoma-advanced esophageal adenocarcinoma sequence. Pathol Res Pract. 213, 205209 (2017).

38. Jeong, G. et al. A Kelch domain-containing KLHDC7B and a long non-coding RNA ST8SIA6-AS1 act oppositely on breast cancer cell proliferation via the interferon signaling pathway. Sci Rep. 8, 12922 (2018).

39. Enomoto, S. et al. Suppression of Slit2/Robo1 mediated HUVEC migration by Robo4. Biochem Biophys Res Commun. 469, 797-802 (2016).

40. Zhou, Y. et al. DHRS2 inhibits cell growth and motility in esophageal squamous cell carcinoma. Oncogene 37, 1086-1094 (2018).

41. Rando, T. A. \& Chang, H. Y. Aging, rejuvenation, and epigenetic reprogramming: resetting the aging clock. Cell 148, 46-57 (2012).

42. Hald, Ø. H., Olsen, L., Gallo-Oller, G., Elfman, L. H. M., Løkke, C. \& Kogner, P. Inhibitors of ribosome biogenesis repress the growth of MYCN-amplified neuroblastoma. Oncogene 38, 2800-2813 (2019).

43. Nishimura, K. et al. Perturbation of ribosome biogenesis drives cells into senescence through 5S RNP-mediated p53 activation. Cell Rep. 10, 1310-1323 (2015).

44. Jiang, Y. et al. LncRNA LINC00460 promotes EMT in head and neck squamous cell carcinoma by facilitating peroxiredoxin-1 into the nucleus. J Exp Clin Cancer Res. 38, 365 (2019).

45. Lamouille, S., Xu, J. \& Derynck, R. Molecular mechanisms of epithelial-mesenchymal transition. Nat Rev Mol Cell Biol. 15, 178-196 (2014).

46. Mannoor, K., Shen, J., Liao, J., Liu, Z. \& Jiang, F. Small nucleolar RNA signatures of lung tumor-initiating cells. Mol Cancer 13, 104 (2014).

47. Gopalan, V., Islam, F. \& Lam, A. K. Surface Markers for the Identification of Cancer Stem Cells. Methods Mol Biol. 1692, 17-29 (2018). 
48. Ofengand, J. \& Bakin, A. Mapping to nucleotide resolution of pseudouridine residues in large subunit ribosomal RNAs from representative eukaryotes, prokaryotes, archaebacteria, mitochondria and chloroplasts. J Mol Biol. 266, 246-268 (1997).

49. Adachi, H., De Zoysa, M. D. \& Yu, Y. T. Post-transcriptional pseudouridylation in mRNA as well as in some major types of noncoding RNAs. Biochim Biophys Acta Gene Regul Mech. 1862, 230-239 (2019).

50. Michel, C. I. et al. Small nucleolar RNAs U32a, U33, and U35a are critical mediators of metabolic stress. Cell Metab. 14, 33-44 (2011).

51. Lee, J. et al. Rpl13a small nucleolar RNAs regulate systemic glucose metabolism. J Clin Invest. 126, 4616-4625 (2016).

52. Brandis, K. A. et al. Box C/D small nucleolar RNA (snoRNA) U60 regulates intracellular cholesterol trafficking. J Biol Chem. 288, 35703-35713 (2013).

53. Jinn, S. et al. snoRNA U17 regulates cellular cholesterol trafficking. Cell Metab. 21, 855867 (2015).

54. Joerger, A. C. \& Fersht, A. R. The p53 Pathway: Origins, Inactivation in Cancer, and Emerging Therapeutic Approaches. Annu Rev Biochem. 85, 375-404 (2016).

55. Riggelen, J. van, Yetil, A. \& Felsher, D. W. MYC as a regulator of ribosome biogenesis and protein synthesis. Nat Rev Cancer 10, 301-309 (2010).

56. Su, H. et al. Elevated snoRNA biogenesis is essential in breast cancer. Oncogene 33, 13481358 (2014).

57. Johnson, D. W., Llop, J. R., Farrell, S. F., Yuan, J., Stolzenburg, L. R. \& Samuelson, A. V. The Caenorhabditis elegans Myc-Mondo/Mad complexes integrate diverse longevity signals. PLoS Genet. 10, e1004278 (2014).

58. Greer, C. et al. Myc-dependent genome instability and lifespan in Drosophila. PLoS One 8, e74641 (2013).

59. Hofmann. J. W. et al. Reduced expression of MYC increases longevity and enhances healthspan. Cell 160, 477-488 (2015).

60. Herter, E. K., Stauch, M., Gallant, M., Wolf, E., Raabe, T. \& Gallant, P. snoRNAs are a novel class of biologically relevant Myc targets. BMC Biol. 13, 25 (2015).

61. Langhendries, J. L., Nicolas, E., Doumont, G., Goldman, S. \& Lafontaine, D. L. The human box C/D snoRNAs U3 and U8 are required for pre-rRNA processing and tumorigenesis. Oncotarget 7, 59519-59534 (2016).

62. Chen, L. et al. SNORD76, a box C/D snoRNA, acts as a tumor suppressor in glioblastoma. Sci Rep. 5, 8588 (2015). 


\section{Figures Legends}

Figure 1) Genomic map, structure, and expression of the human snoRNA-jouvence.

A) Genomic map of the snoRNA-jouvence. $h$-jou is localized on chromosome 11 (GRCh38.p13) in a large intron of the gene TEAD1. B) Secondary structure of the $h$-jou, as determined by the UNAFold software (http://unafold.rna.albany.edu/?q=DINAMelt/software). As predicted by its primary sequence, and like its Drosophila orthologue, $h$-jou is a H/ACA box forming a double hairpin. C) Expression level (1/dCt x 100) (dCT= Ct snoRNA - Ct GAPDH) in various cell lines determined by RT-qPCR (Taqman). Compared to the standard reference gene GAPDH, $h$-jou is weakly expressed in all different tested cell lines, with a very weak expression (to the limit of detection) in the U87 line (glioblastoma), and the strongest expression in the HEK293 cells (human embryonic kidney).

Figure 2) The overexpression of $h$-jou increases the proliferation of the cells.

A) Cells number of HCT116 stably transfected cells with jouvence (in plasmid pcDNA-3.1) compared to the empty vector transfected cells determined by VicellXR. B) Same batch of cells than A, but spread and grow in 96 cell-plates and evaluated by luminescence (CellTiter-Glo), which quantify the amount of ATP, and consequently but indirectly, the number of cells. C) Cells number of HEK293 stably transfected cells with jouvence compared to the empty vector transfected cells determined by VicellXR. D) Same batch of cells than D, but spread and growth in 96 cell-plates and evaluated by luminescence (CellTiter-Glo). E) Cells number of Caco-2 stably transfected cells with jouvence compared to the empty vector transfected cells determined by VicellXR. The cells overexpressing jouvence are more numerous after 24 hours post-seeding, which have doubled their number after 72 hours, and again, more than tripled after 160h. F-G-H) Expression level (Fold change) of the transfected snoRNA-jouvence (overexpression) compared to non-transfected cells. F) In HCT116, $h$-jou is increased by about 350 -fold. G) In Caco-2, the expression level of $h$-jou is increased by about 200 -fold. H) In HEK293, the expression level is increased by about 1200-fold. Statistics: For the VicelXR (AC-D) $n=2$, for the Luminescence CellTiter-Glo (B-D), $n=6$. Each figure is representative of three independent experiments. (p-values: $* \mathrm{p}<0,05 ; * * \mathrm{p}<0,005 ; * * * \mathrm{p}<0,0005)$. Errors bars represent the mean + /- S.E.M. (p-value were calculated using the one-tail unpaired t-test using GraphPad Prism). 


\section{Figure 3) The overexpression of jou by lentivirus increases cells proliferation.}

A) Cells number of HCT116 cells transduced with jou-lentivirus, at two different MOI (1 and 10) compared to non-transduced cells (NT) determined by VicellXR. B) Same batch of cells than A, but spread and grow in 96 cell-plates and evaluated by luminescence (CellTiter-Glo), which quantify the amount of ATP, and consequently but indirectly, the number of cells. Similarly, the amount of luminescence is increased after 96 hours for the two tested MOI. C) Expression level (Fold change) of the transduced $h$-jou (overexpression) compared to nontransduced cells. In HCT116, $h$-jou is increased by about 11-fold for the MOI-1, and 14-fold for MOI-10. D) Cells number of Caco-2 cells transduced with jou-lentivirus (LV), at MOI-10 compared to non-transduced cells (NT) determined by VicellXR. E) Same batch of cells than D, but spread and grow in 96 cell-plates and evaluated by luminescence (CellTiter-Glo). F) Cells number of RPE-1 cells transduced with jou-lentivirus (LV), at MOI-10 compared to nontransduced cells (NT) determined by VicellXR. G) Same batch of cells than F, but spread and grow in 96 cell-plates and evaluated by luminescence (CellTiter-Glo). H) Cells number of HUVEC transduced with jou-lentivirus (LV) at MOI-10 and MOI-20 compared to nontransduced cells (NT), spread and grow in 96 cell-plates and evaluated by luminescence (CellTiter-Glo). The amount of luminescence is increased after 96 hours. Statistics: For the VicelXR (A-D-F) $n=3$, for the Luminescence CellTiter-Glo (B-E-G-H), n=10. Each figure is representative of two independent experiments. ( $\mathrm{p}$-values: * $\mathrm{p}<0,05$; ** $\mathrm{p}<0,005$; *** $\mathrm{p}<0,0005$ ). Errors bars represent the mean +/- S.E.M. (p-value were calculated using the onetail unpaired t-test using GraphPad Prism).

\section{Figure 4) The knockdown of $h$-jou by si-RNA decreases the proliferation.}

A) Cells number of HCT116 transiently transfected cells with LNA-siRNA directed against jouvence compared to the control non-transfected cells (Co = treated with the same amount of RNAiMAX), and to the control ( $\mathrm{Si}-\mathrm{Co}=$ transfected cells with a siRNA-control without target), determined by VicellXR 72 hours post-seeding. B) The expression level (Fold change) of the siRNA transfected HCT116 cells compared to the non-transfected cells, determined 72 hours post-seeding (same batch of cells than A). C) Cells number of MCF7 transiently transfected cells compared to their two respective controls, as in A, determined by VicellXR. D) Same batch of cells than $\mathrm{C}$, but spread and growth in 96 cell-plates and evaluated by luminescence (CellTiter-Glo) after 96 hours. E) Cells number of U87 transiently transfected cells with LNAsiRNA compared to their respective controls, determined by VicellXR. F) Same batch of cells 
than E, but spread and growth in 96 cell-plates and evaluated by luminescence after 96 hours. G) Cells number of A549 transiently transfected cells with LNA-siRNA compared to their control, determined by VicellXR. H) Same batch of cells than G, but spread and growth in 96 cell-plates and evaluated by luminescence after 96 hours. I) Cells number of HUVEC (primary cells) transiently transfected cells with LNA-siRNA compared to their respective control, determined by VicellXR. J) Same batch of cells than I, but spread and growth in 96 cell-plates and evaluated by luminescence. Statistics: For the VicelXR (A-C-E-G-I) $n=3$, for the Luminescence CellTiter-Glo (B-D: $n=27),(H: n=8),(J: n=10)$. Each figure is representative of three independent experiments. (p-values: $* \mathrm{p}<0,05 ; * * \mathrm{p}<0,005 ; * * * \mathrm{p}<0,0005$ ). Errors bars represent the mean +/- S.E.M. (p-value were calculated using the one-tail unpaired t-test using GraphPad Prism).

\section{Figure 5) Hundreds of genes are deregulated in HCT116 cells overexpressing jouvence}

A) Transcriptomic analysis (RNA-Seq) performed on total-RNA from the overexpressing jouvence HCT116 cells compared to the empty vector cells, reveals that 5918 genes are deregulated, in which 2974 are upregulated, while 2944 are downregulated (see Suppl. TableS1 and Suppl. Table-S2 for the complete list of genes). B) Clusters analysis (Heat map) of differentially expressed genes. C) Statistic of enrichment pathway of the deregulated genes according to the KEGG analysis (see Suppl. Table S3) for the full list of KEGG analysis. In brief, the metabolic pathways are the main deregulated pathways in term of number of genes, while the ribosome is the main deregulated pathway in term of strength (Rich factor).

\section{Figure 6) Hundreds of genes are deregulated in jouvence depleted HCT116 cells.}

A) Transcriptomic analysis (RNA-Seq) performed on total-RNA from the knockdown of jouvence by siRNA on HCT116 cells compared to the control non-transfected cells, reveals that 6263 genes are deregulated, in which 3098 are upregulated, while 3165 are downregulated (see Suppl. Table-S4 and Suppl. Table-S5 for the complete list of genes). B) Clusters analysis (Heat map) of differentially expressed genes. C) Statistic of pathway enrichment of the deregulated genes according to the KEGG analysis (see Suppl. Tables S6 for the full list of the KEGG analysis). In brief, the metabolic pathways are the main deregulated pathways in term of number of genes, while the ribosome and spliceosome are the two main deregulated pathways in term of strength (Rich factor). 


\section{Figure 7) Summary of main deregulated genes suggesting a dedifferentiation.}

Several of the main key Transcription Factors genes (Twist, Snail, ZEB) involved in EMT are upregulated in jou-overexpression. Consequently, several of their known targets as $\mathrm{N}$-cadherin, Fibronectin, few Claudin and several KLF are upregulated. In parallel several genes are downregulated, as E-cadherin (CDH1), Claudin, $\alpha$-catenin, $\beta$-catenin, several Annexin, several Keratin and several Myosin, as well as Protocadherin1- $\beta 13, \alpha \mathrm{C} 1, \gamma 9$. Inversely, in the joudepleted cells by siRNA, we rather observe the opposite phenotype, suggesting a MET (Mesenchymal-Epithelial-Transition). More particularly, we observe a decrease of two Transcription Factors as SOX4 and SOX8, although these two last have not yet been clearly demonstrated to induce MET. Several other genes (putatively their targets) are upregulated, while two key genes, as EPCAM and CD44 are downregulated. This last group of four genes (blue ellipse) could also suggests a CSC phenotype. Nevertheless, although we do not observe all the characteristic cells markers of the EMT, several of the deregulated genes in jouoverexpression strongly suggest an EMT, or at least a partial or hybrid EMT, as suggested by Pastushenko and Blanpain (2019) [18], while inversely, the knock-down of jouvence rather seems to direct the cells toward a MT.

Table 1) Short list of the main genes deregulated in overexpressing jouvence HCT116 cells revealed by RNA-Seq.

A) Up-regulated genes. B) Down-regulated genes. C) Short list of the main deregulated pathways revealed by the KEGG analysis. For the complete list, see Suppl. Tables S1-S2-S3 respectively.

Table 2) Short list of the main genes deregulated in knockdown jouvence HCT116 cells revealed by RNA-Seq.

A) Up-regulated genes. B) Down-regulated genes. C) The main deregulated pathways revealed by the KEGG analysis. For the complete list, see Suppl. Tables S4-S5-S6 respectively. 


\section{Supplementary Information}

\section{Suppl. Figure 1) Validation by RT-qPCR of few selected deregulated genes in jou overexpression or knockdown.}

A) RT-qPCR (SybGreen) results of the quantification of few selected genes in jou overexpressing HCT116 cells. Fold change comparing HCT116 transfected cells versus empty plasmid cells. As revealed by the RNA-Seq, the genes BCL2, CLDN9, CTNNAL, and HNF4A are downregulated, while the genes CTGF, HDAC5, IGF1R, SNAI3, IRS2, and SOX4 are upregulated. B) RT-qPCR (SybGreen) results of the quantification of few selected genes in jou knockdown. Fold change comparing HCT116 siRNA transfected cells versus non-transfected cells. FDFT1, HMGCR, PRPF, SRSF4 are downregulated, while CLDN2, DHRS2, ILORB, and SLC7A11 genes are upregulated $(\mathrm{n}=2)$. The RT-qPCR confirms the deregulation of those genes revealed by RNA-Seq.

\section{Supplementary Tables S1 to S8}

Eight Supplementary Tables (S1 to S8) containing the RNA-Seq Data-analysis (Excel files).

Table S1-HCT-jou-overexpression_DEG upregulated genes.xlsx

Table S2-HCT-jou-overexpression_DEG downregulated genes.xlsx

Table S3-HCT-jou-overexpression_KEGG enrichment pathways.xlsx

Table S4-HCT-siRNA knockdown_DEG upregulated genes.xlsx

Table S5-HCT-siRNA knockdown_DEG downregulated genes.xlsx

Table S6-HCT-siRNA knockdown_KEGG enrichment pathways.xlsx

Table S7-jou-overexpression-genes Up vs siRNA-genes Down-1102 common genes.xlsx

Table S8-jou-overexpression-genes Down vs siRNA-genes Up-868 common genes.xlsx 
El Khoury et al.,

Figure 1)

A)

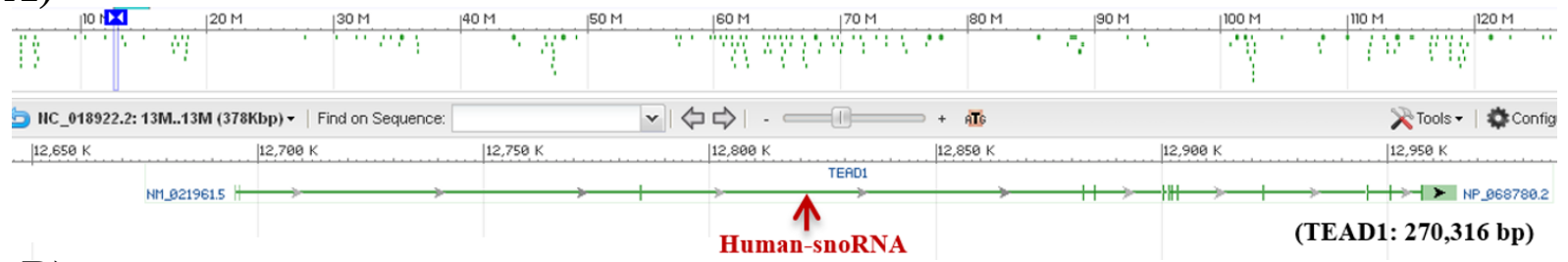

B)

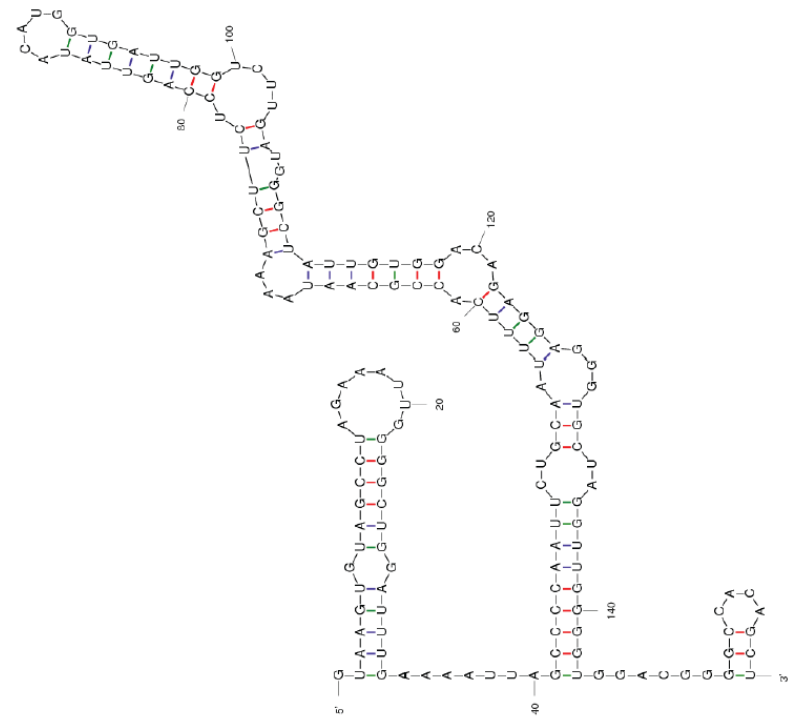

C)

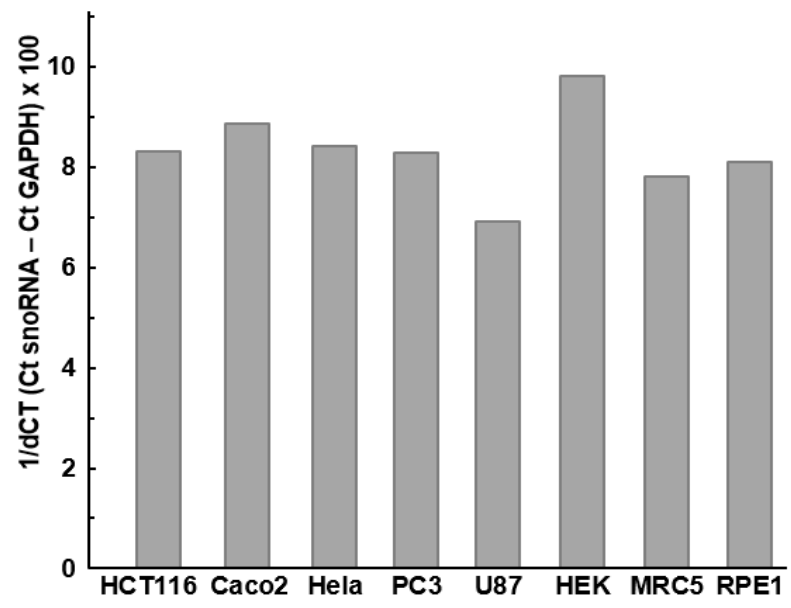


A)

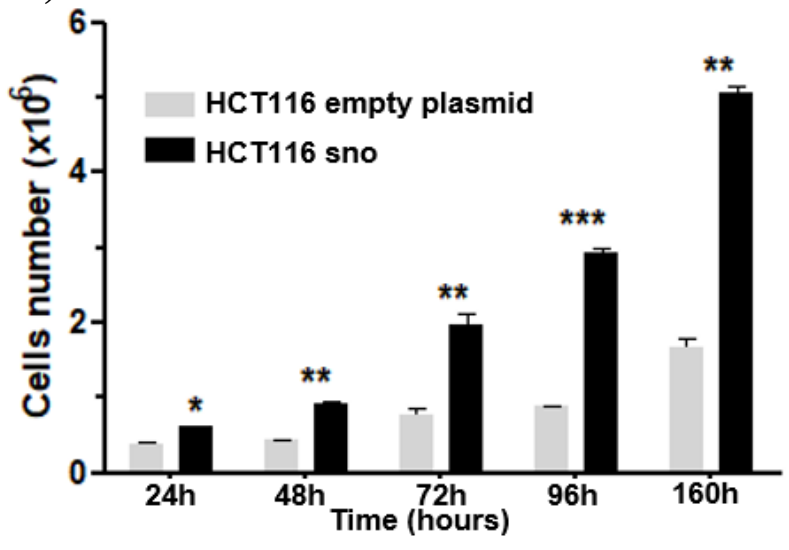

C)

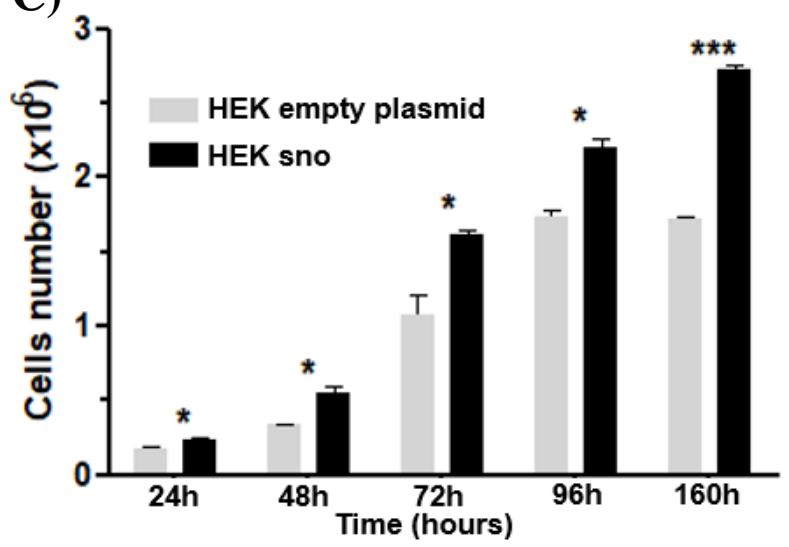

E)

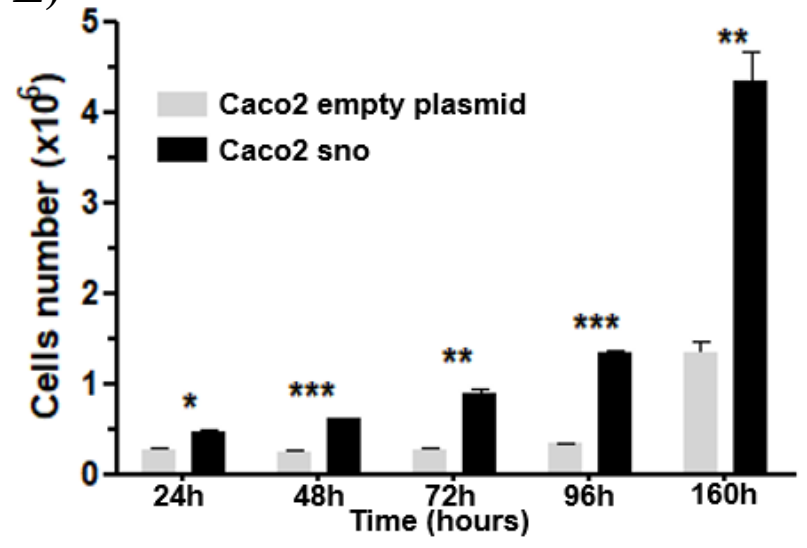

B)

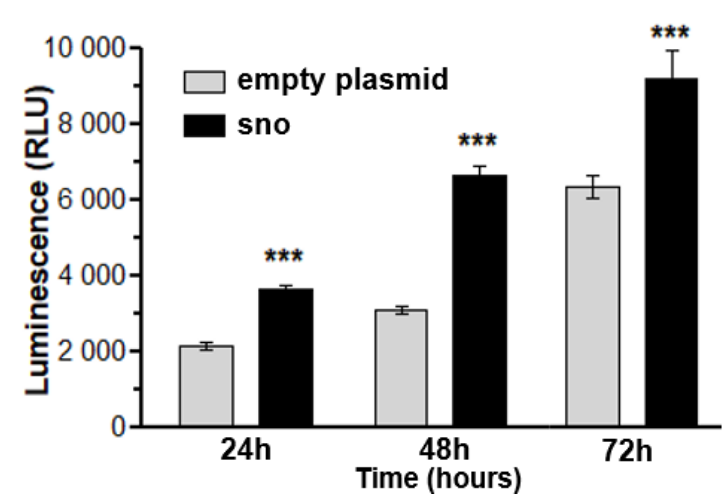

D)

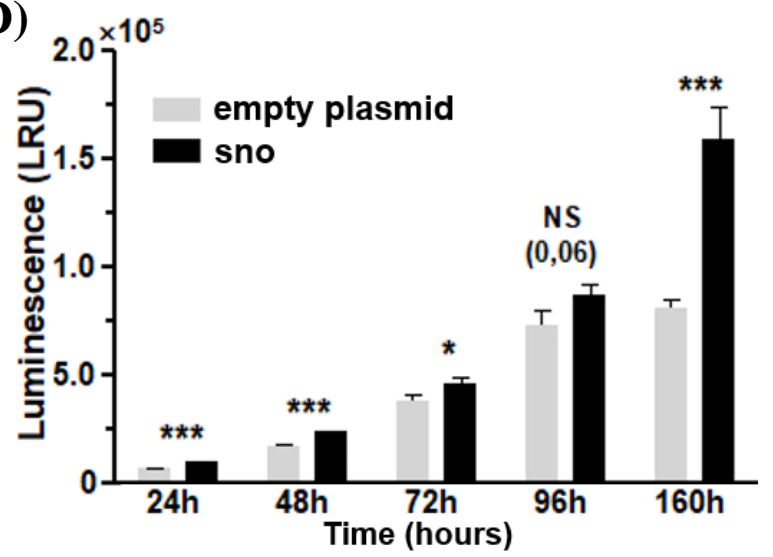

F)

G)

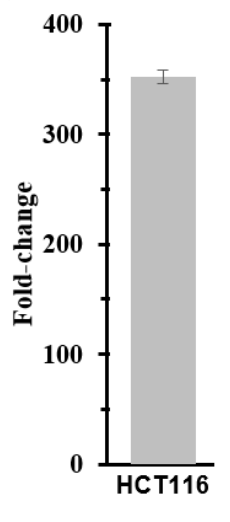

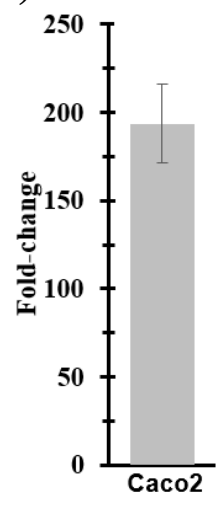

H)

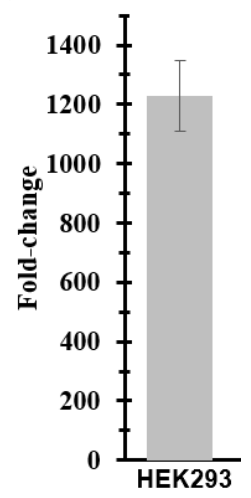


Figure 3)

El Khoury et al.,

A)

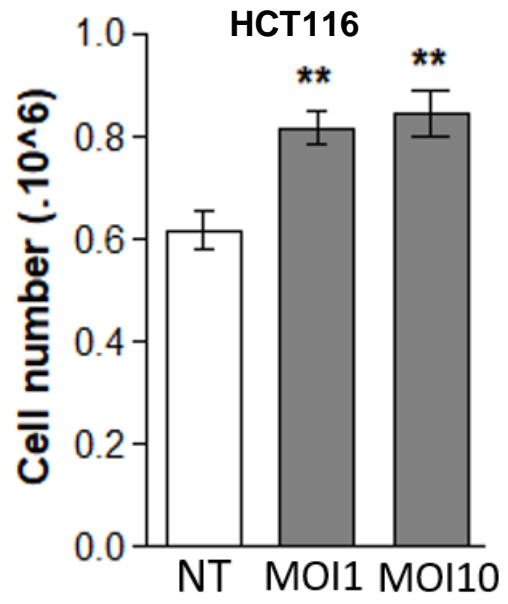

B)

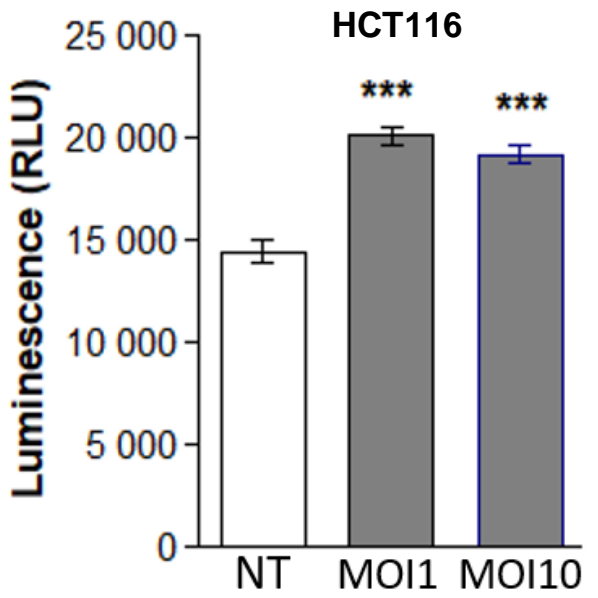

C)

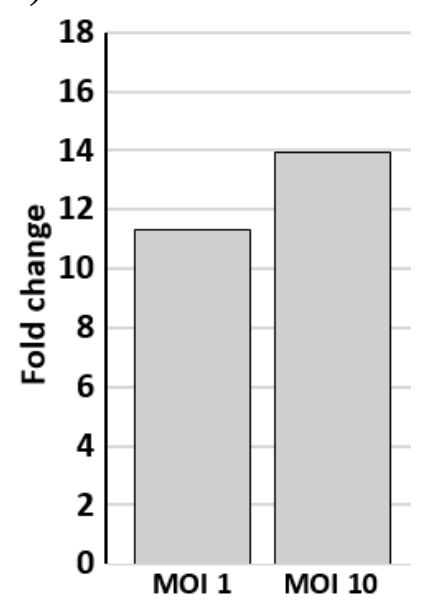

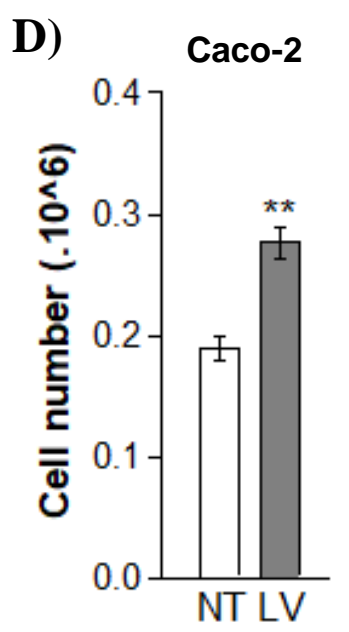

E) $\mathrm{Caco}-2$

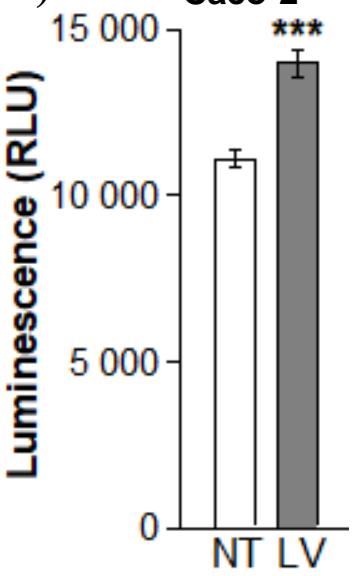

F) 0.4 RPE-1

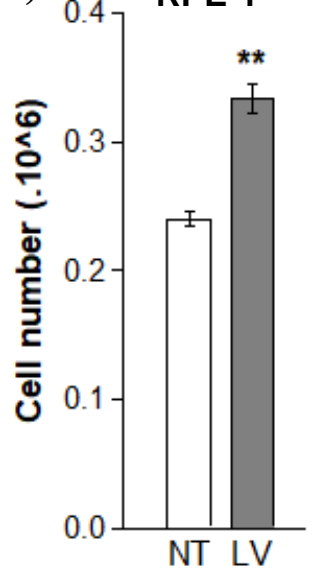

G) RPE-1 ***

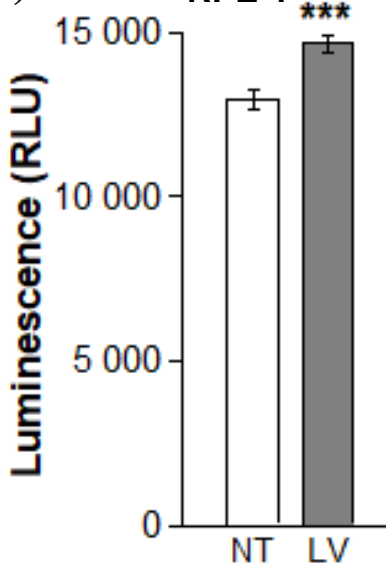

H)

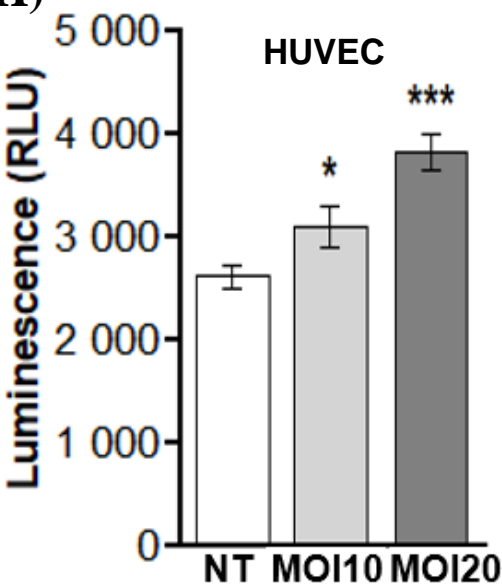


Figure 4)
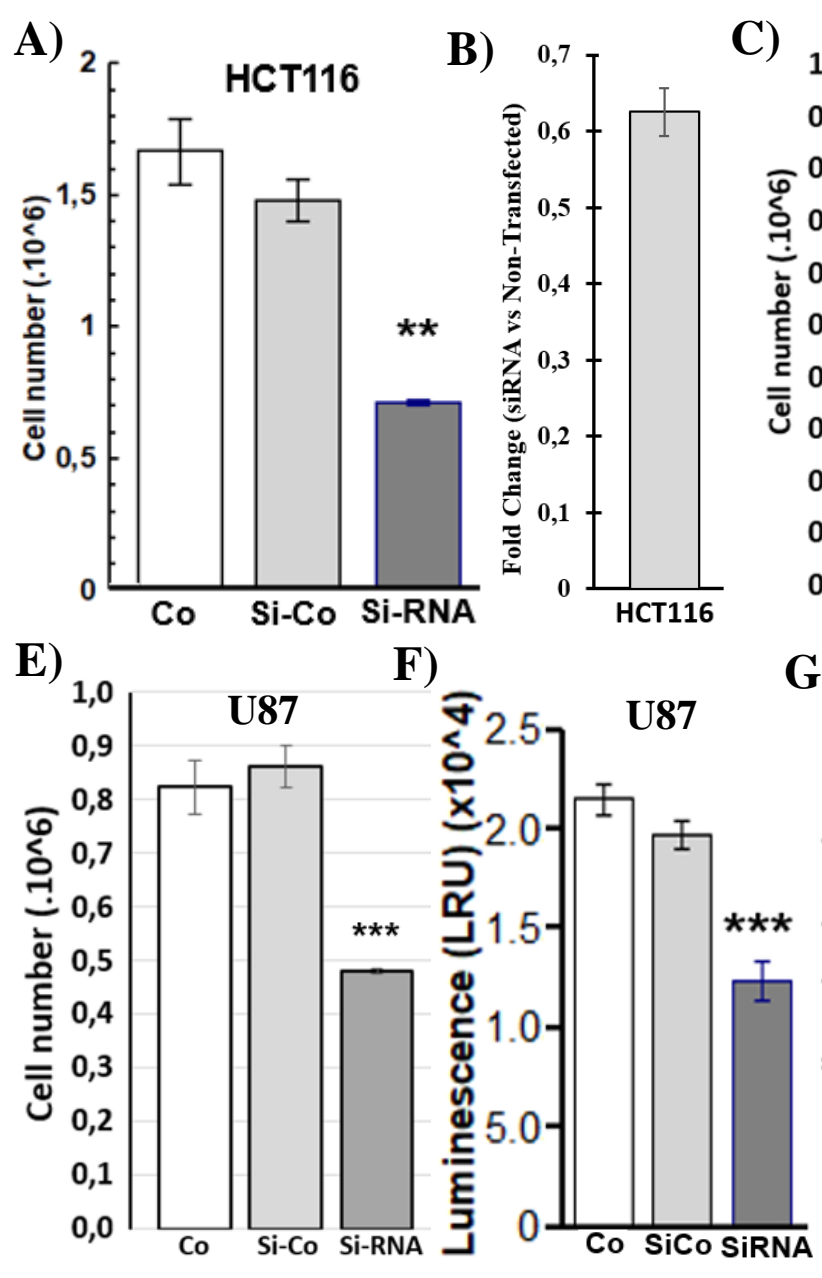

El Khoury et al.,
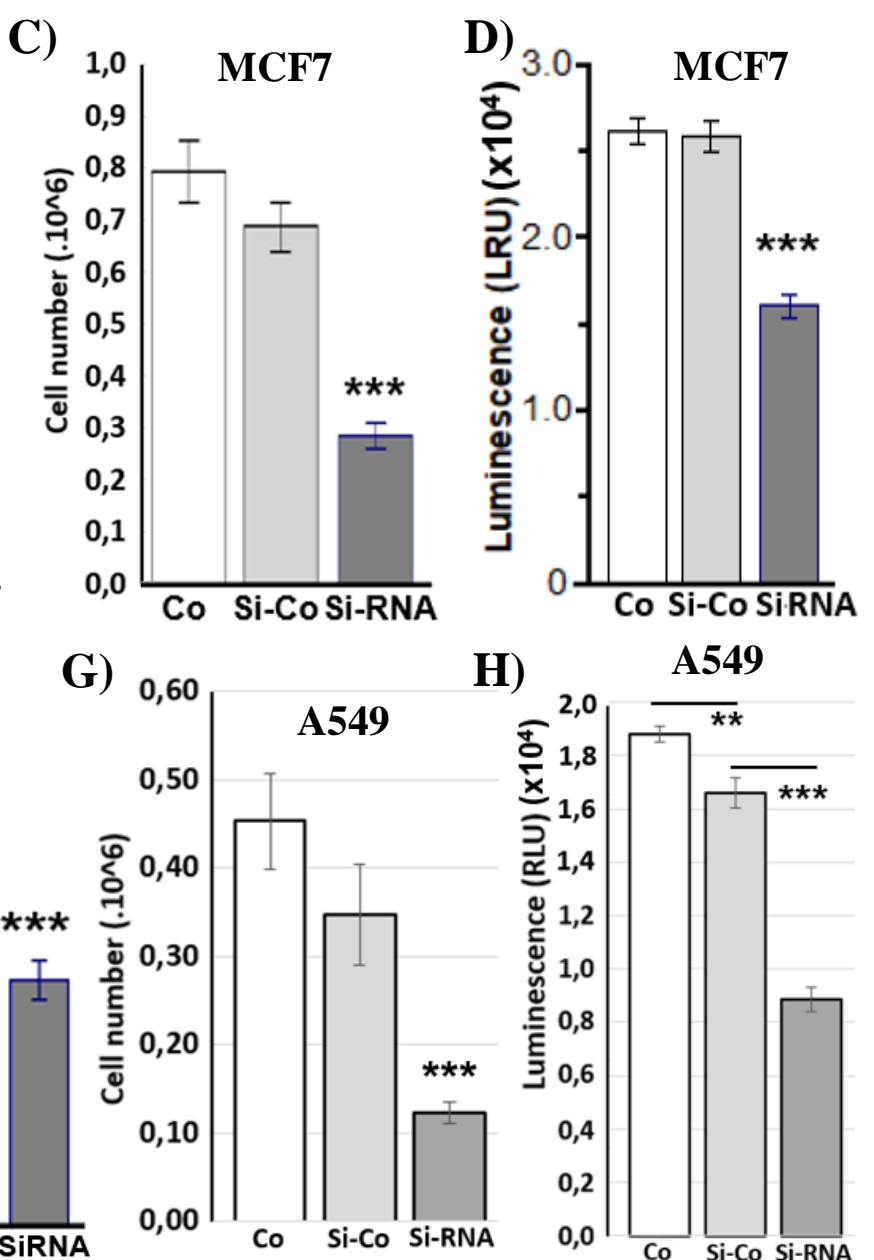

H) $\quad \mathbf{A 5 4 9}$

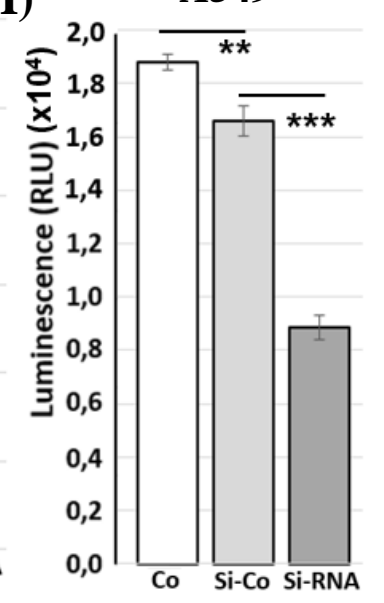

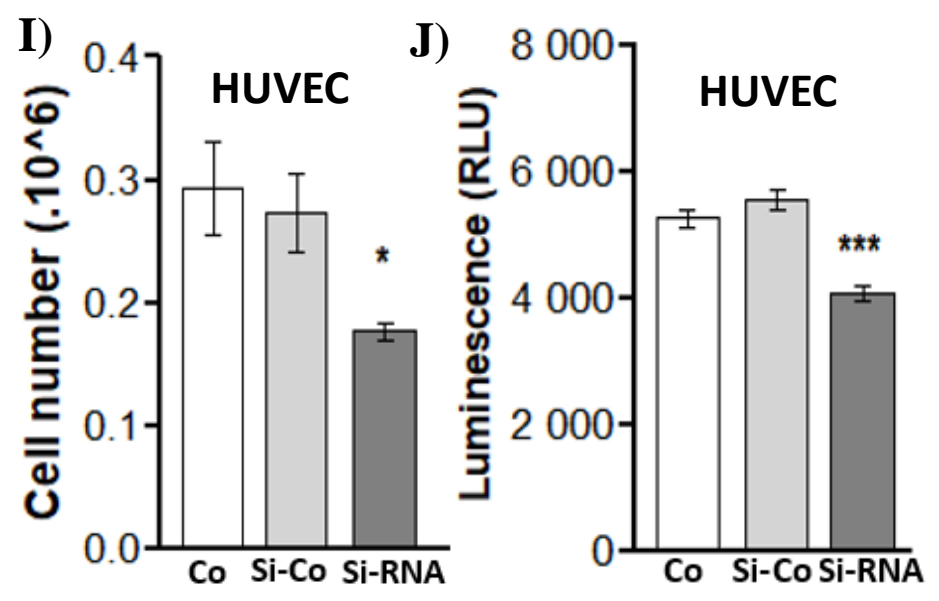




\section{Figure 5)}

A) Differential Expressed Genes ( 5918) HCT_sno vs HCT_vec
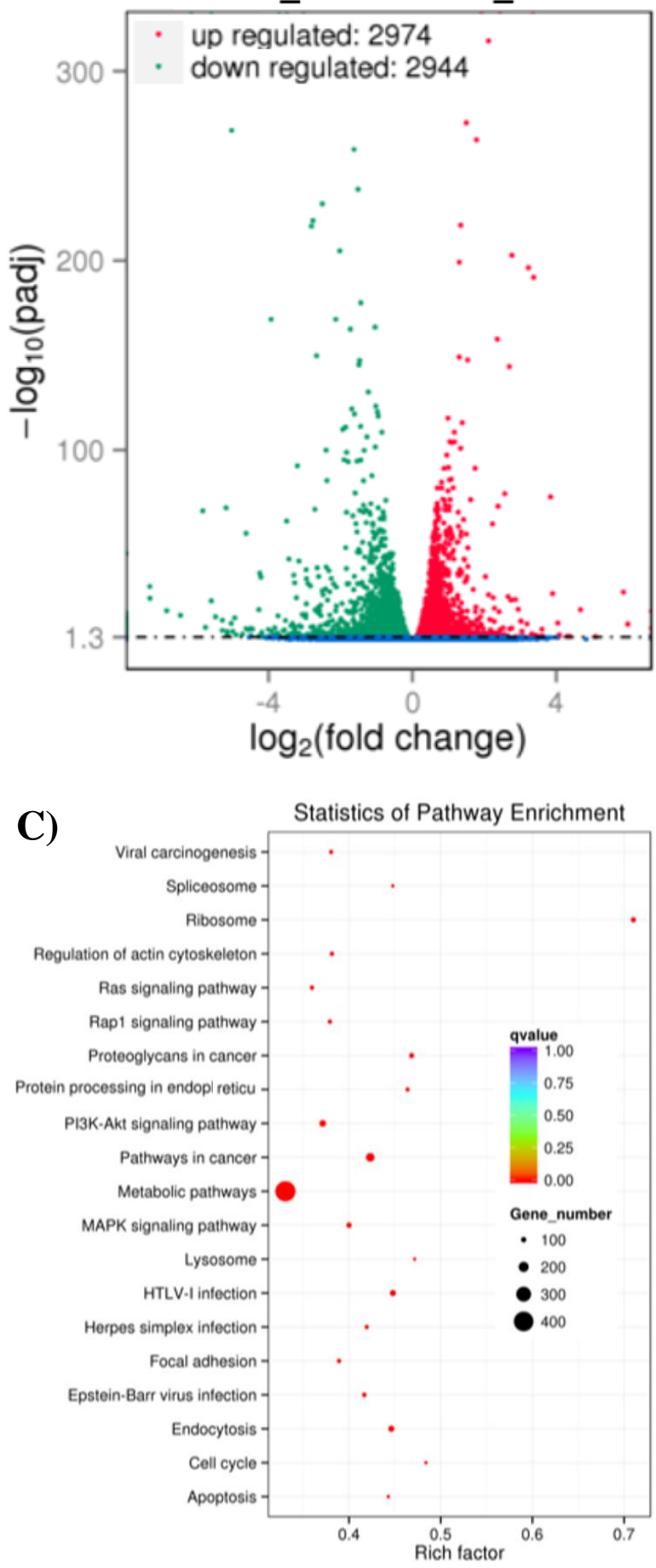

El Khoury et al.,

B)

Cluster analysis of differentially expressed genes

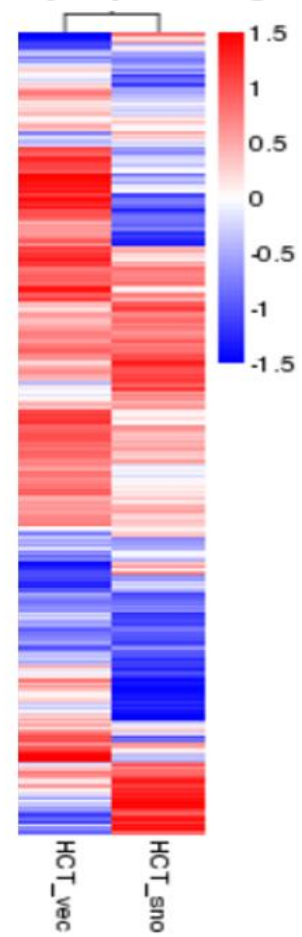


A)

Differential Expressed Genes ( 6263 ) HCT_siRNA vs HCT_NT

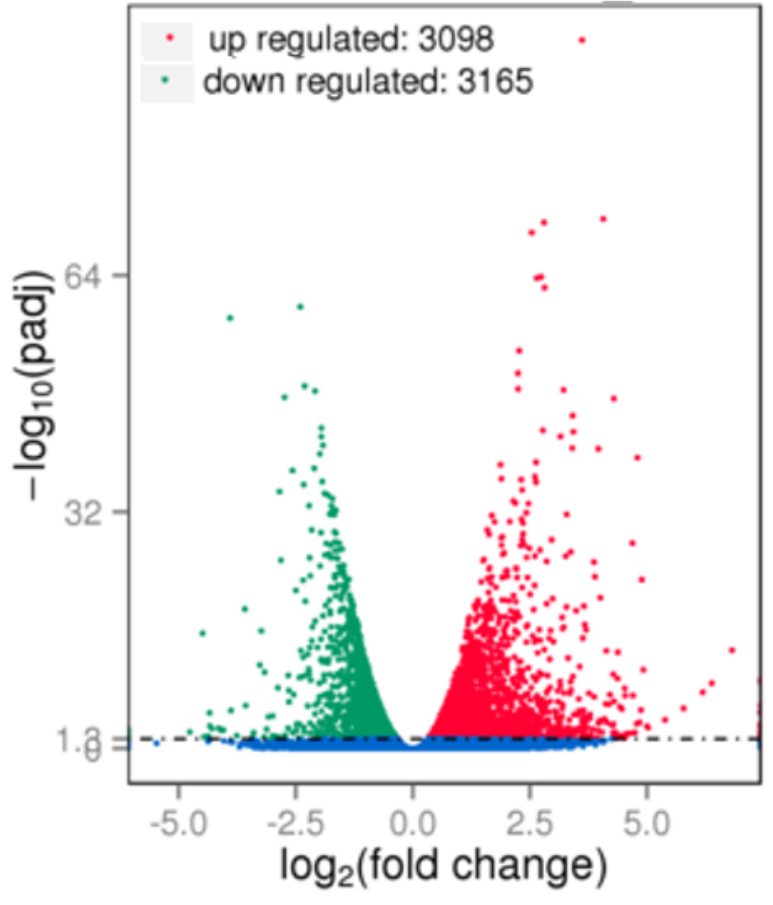

C)

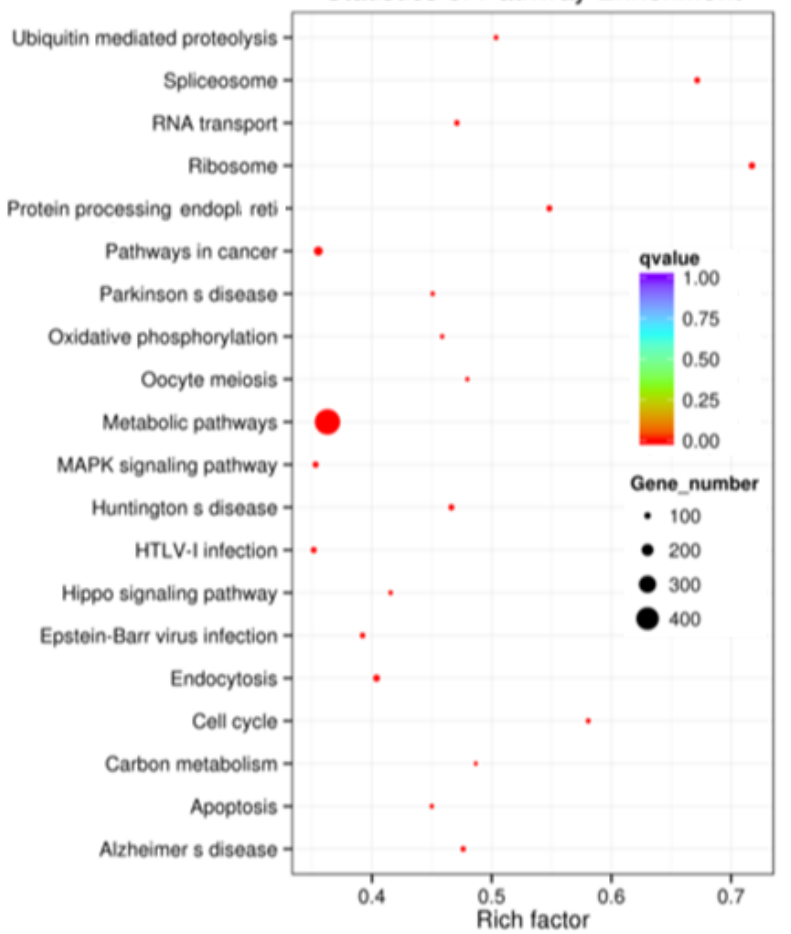

Statistics of Pathway Enrichment
B)
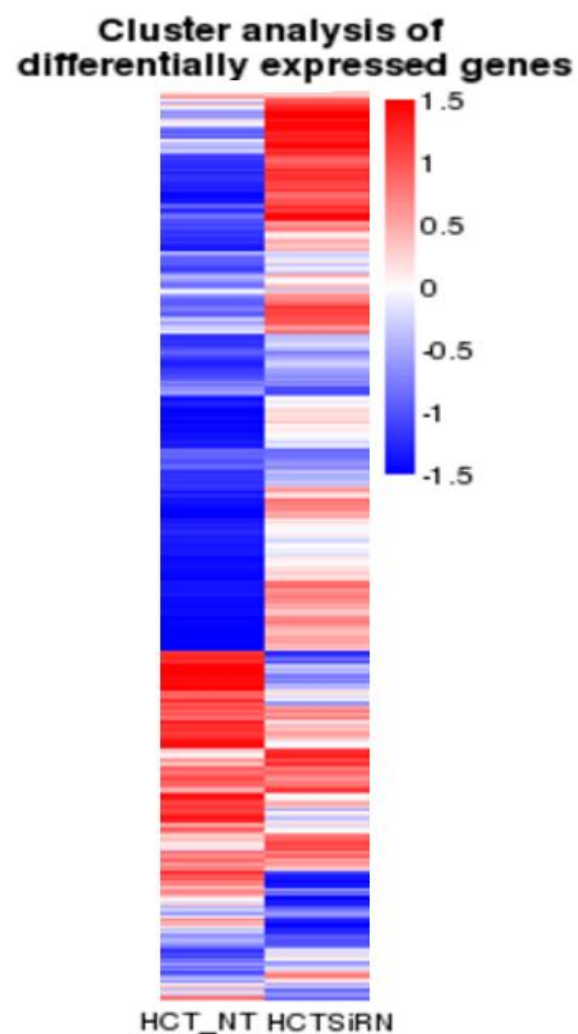
Figure 7)

El Khoury et al.,

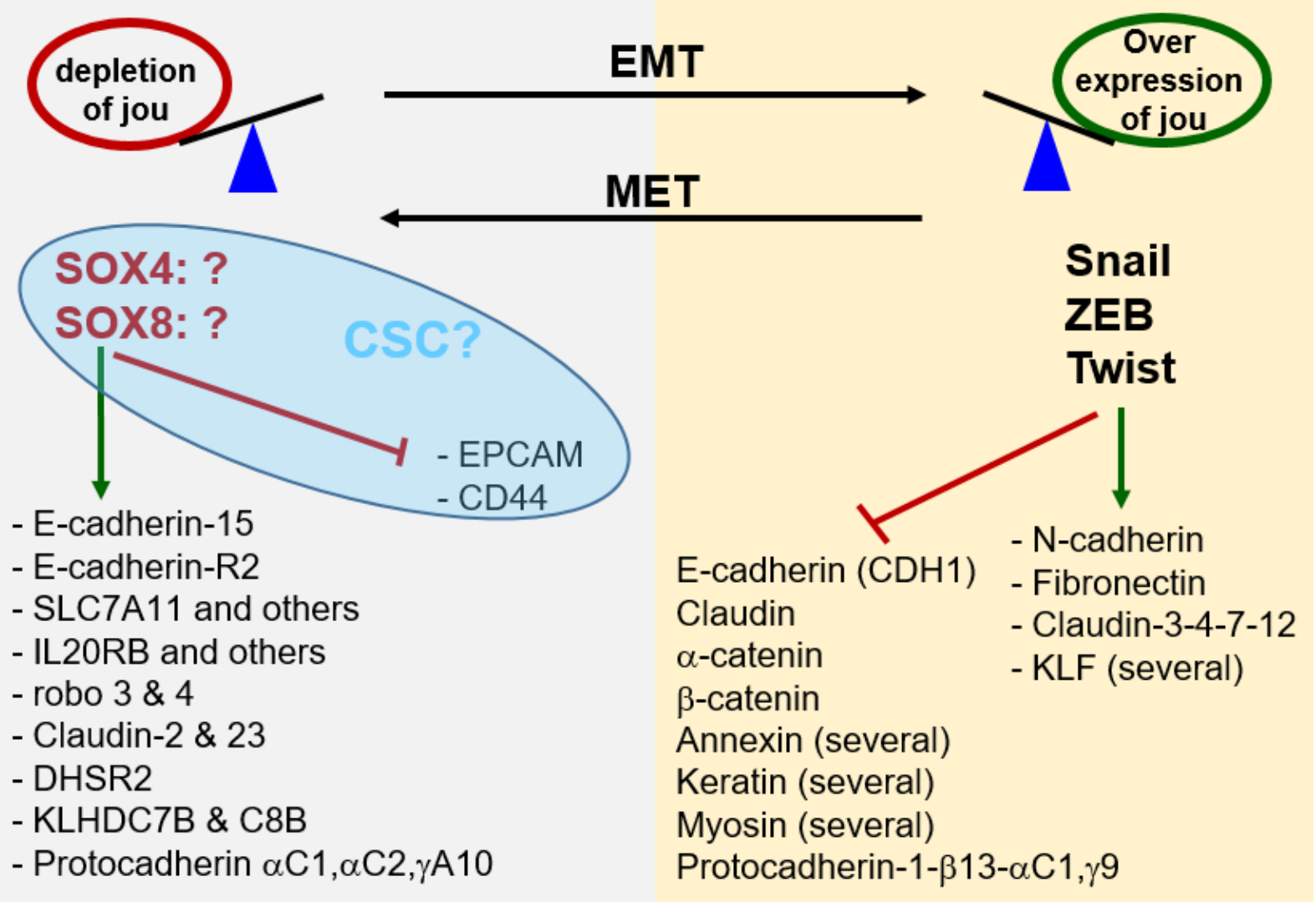


bioRxiv preprint doi: https://doi.org/10.1101/2020.06.17.157321; this version posted June 18, 2020. The copyright holder for this preprint (which was not certified by peer review) is the author/funder. All rights reserved. No reuse allowed without permission.

\begin{tabular}{|c|c|c|c|c|}
\hline Table 1) Gene_id & Fold Change & padj & Gene Name & Description \\
\hline \multicolumn{5}{|c|}{ A) HCT116-jou-overexpression (Up-regulated genes) } \\
\hline ENSG00000124766 & 2,82 & $1,86 \mathrm{E}-273$ & SOX4 & SRY(sex determ region Y)-box 4 \\
\hline ENSG00000128564 & 3,43 & $1,93 \mathrm{E}-264$ & VGF & VGF nerve growth factor inducible \\
\hline ENSG00000049130 & 2,54 & $2,33 \mathrm{E}-219$ & KITLG & KIT ligand \\
\hline ENSG00000175745 & 6,80 & $1,64 \mathrm{E}-203$ & NR2F1 & nuclear receptor subfamily 2 group $F$ \\
\hline ENSG00000158710 & 2,47 & $6,78 \mathrm{E}-200$ & TAGLN2 & transgelin 2 \\
\hline ENSG00000089472 & 9,35 & $6,17 \mathrm{E}-197$ & HEPH & hephaestin \\
\hline ENSG00000077274 & 10,32 & $7,35 \mathrm{E}-192$ & CAPN6 & calpain 6 \\
\hline ENSG00000079215 & 5,15 & $2,63 \mathrm{E}-159$ & SLC1A3 & solute carrier family 1 (glial affinity) \\
\hline ENSG00000127528 & 2,46 & $7,89 \mathrm{E}-150$ & KLF2 & Kruppel-like factor 2 (lung) \\
\hline ENSG00000146376 & 2,89 & $3,19 \mathrm{E}-148$ & ARHGAP18 & Rho GTPase activating protein 18 \\
\hline ENSG00000165949 & 6,46 & $9,82 \mathrm{E}-145$ & IFI27 & interferon, alpha-inducible protein 27 \\
\hline ENSG00000138434 & 1,98 & $1,37 \mathrm{E}-117$ & SSFA2 & sperm specific antigen 2 \\
\hline ENSG00000120738 & 2,61 & $3,23 \mathrm{E}-115$ & EGR1 & early growth response 1 \\
\hline ENSG00000204574 & 2,24 & $3,36 \mathrm{E}-110$ & $\mathrm{ABCF} 1$ & ATP-binding cassette, sub-family F \\
\hline ENSG00000091136 & 2,06 & $4,04 \mathrm{E}-105$ & LAMB1 & laminin, beta 1 \\
\hline ENSG00000179094 & 2,25 & $5,37 \mathrm{E}-105$ & PER1 & period circadian clock 1 \\
\hline ENSG00000140450 & 2,13 & $8,38 \mathrm{E}-105$ & ARRDC4 & arrestin domain containing 4 \\
\hline ENSG00000115457 & 2,53 & $1,12 \mathrm{E}-101$ & IGFBP2 & insulin-like growth factor bind. prot2 \\
\hline ENSG00000105373 & 1,94 & $4,02 \mathrm{E}-98$ & GLTSCR2 & glioma tumor suppressor \\
\hline \multicolumn{5}{|c|}{ B) HCT116-jou-overexpression (Down-regulated genes) } \\
\hline ENSG00000267761 & 0,03 & $1,91 \mathrm{E}-269$ & CTD-2130O13.1 & - \\
\hline ENSG00000142910 & 0,32 & $2,10 \mathrm{E}-259$ & TINAGL1 & tubulointerstitial nephritis antigen-like \\
\hline ENSG00000101188 & 0,35 & $2,04 \mathrm{E}-238$ & NTSR1 & neurotensin receptor 1 (high affinity) \\
\hline ENSG00000130508 & 0,18 & $1,08 \mathrm{E}-230$ & PXDN & peroxidasin homolog (Drosophila) \\
\hline ENSG00000169035 & 0,15 & $6,81 \mathrm{E}-222$ & KLK7 & kallikrein-related peptidase 7 \\
\hline ENSG00000099994 & 0,14 & $6,04 \mathrm{E}-219$ & SUSD2 & sushi domain containing 2 \\
\hline ENSG00000175906 & 0,25 & $7,80 \mathrm{E}-206$ & ARL4D & ADP-ribosylation factor-like 4D \\
\hline ENSG00000135074 & 0,37 & $1,79 \mathrm{E}-178$ & ADAM19 & ADAM metallopeptidase domain 19 \\
\hline ENSG00000103044 & 0,23 & $9,15 \mathrm{E}-170$ & HAS3 & hyaluronan synthase 3 \\
\hline ENSG00000108244 & 0,07 & $9,31 \mathrm{E}-170$ & KRT23 & keratin 23 (histone deacet. inducible) \\
\hline ENSG00000124762 & 0,49 & $1,36 \mathrm{E}-165$ & CDKN1A & cyclin-dependent kinase inhibitor $1 \mathrm{~A}$ \\
\hline ENSG00000101311 & 0,30 & $1,40 \mathrm{E}-164$ & FERMT1 & fermitin family member 1 \\
\hline ENSG00000089356 & 0,16 & $1,72 \mathrm{E}-150$ & FXYD3 & FXYD domain cont. ion transport \\
\hline ENSG00000197081 & 0,36 & $4,84 \mathrm{E}-148$ & IGF2R & insulin-like growth factor 2 receptor \\
\hline ENSG00000167779 & 0,36 & $1,08 \mathrm{E}-145$ & IGFBP6 & insulin-like growth factor bind. prot6 \\
\hline ENSG00000180921 & 0,43 & $2,15 \mathrm{E}-131$ & FAM83H & family with sequence similarity 83 \\
\hline ENSG00000117394 & 0,49 & $6,20 \mathrm{E}-124$ & SLC2A1 & solute carrier family 2 \\
\hline ENSG00000148346 & 0,31 & $1,58 \mathrm{E}-122$ & LCN2 & lipocalin 2 \\
\hline ENSG00000130513 & 0,51 & $5,91 \mathrm{E}-121$ & GDF15 & growth differentiation factor 15 \\
\hline
\end{tabular}

C) HCT116-jou-overexpression (KEGG enrichment pathways)

\#Term (KEGG pathway)

Ribosome

Metabolic pathways

Pathways in cancer

Endocytosis

Spliceosome

Adherens junction

HTLV-I infection

MAPK signaling pathway

Herpes simplex infection

RNA transport

Epstein-Barr virus infection

Tight junction

Ras signaling pathway

Wnt signaling pathway

Huntington's disease

Proteoglycans in cancer

Ribosome biogenesis in eukaryote:

Protein processing in endoplasmic

Axon guidance

ID

hsa03010

Input

hsa01100

hsa05200

hsa04144

hsa03040

hsa04520

hsa05166

hsa04010

hsa05168

hsa03013

hsa05169

hsa04530

hsa04014

hsa04310

hsa05016

hsa05205

hsa03008

hsa04141

hsa04360

$\begin{array}{ccc}89 & 138 & 3,06 \mathrm{E}-42 \\ 189 & 1243 & 8,03 \mathrm{E}-17 \\ 75 & 397 & 4,62 \mathrm{E}-10 \\ 57 & 260 & 8,96 \mathrm{E}-10 \\ 36 & 134 & 4,40 \mathrm{E}-08 \\ 26 & 74 & 8,45 \mathrm{E}-08 \\ 51 & 259 & 1,47 \mathrm{E}-07 \\ 50 & 255 & 2,06 \mathrm{E}-07 \\ 41 & 186 & 2,50 \mathrm{E}-07 \\ 38 & 172 & 7,33 \mathrm{E}-07 \\ 42 & 204 & 7,33 \mathrm{E}-07 \\ 32 & 139 & 3,40 \mathrm{E}-06 \\ 42 & 228 & 8,83 \mathrm{E}-06 \\ 31 & 143 & 1,39 \mathrm{E}-05 \\ 37 & 193 & 1,60 \mathrm{E}-05 \\ 38 & 205 & 2,19 \mathrm{E}-05 \\ 23 & 89 & 2,50 \mathrm{E}-05 \\ 33 & 166 & 2,57 \mathrm{E}-05 \\ 34 & 176 & 2,97 \mathrm{E}-05\end{array}$


bioRxiv preprint doi: https://doi.org/10.1101/2020.06.17.157321; this version posted June 18, 2020. The copyright holder for this preprint (which was not certified by peer review) is the author/funder. All rights reserved. No reuse allowed without permission.

\begin{tabular}{|c|c|c|c|c|}
\hline Table 2) Gene_id & Fold Change & padj & Gene Name & Description \\
\hline \multicolumn{5}{|c|}{ A) HCT116-jou-knockdown (siRNA) (Up-regulated genes) } \\
\hline ENSG00000100867 & 12,26 & $1,51 \mathrm{E}-96$ & DHRS2 & dehydrogenase/reductase (SDR) \\
\hline ENSG00000151012 & 16,74 & $2,25 \mathrm{E}-72$ & SLC7A11 & solute carrier family 7 \\
\hline ENSG00000164171 & 6,97 & $6,83 \mathrm{E}-72$ & ITGA2 & integrin, alpha 2 (CD49B) \\
\hline ENSG00000070669 & 5,81 & $1,65 \mathrm{E}-70$ & ASNS & asparagine synthetase \\
\hline ENSG00000166750 & 6,68 & $1,62 \mathrm{E}-64$ & SLFN5 & schlafen family member 5 \\
\hline ENSG00000101311 & 6,26 & $2,36 \mathrm{E}-64$ & FERMT1 & fermitin family member 1 \\
\hline ENSG00000205730 & 7,02 & $4,60 \mathrm{E}-63$ & ITPRIPL2 & inositol 1,4,5-trisphosphate recep. \\
\hline ENSG00000149948 & 4,82 & $1,58 \mathrm{E}-54$ & HMGA2 & high mobility group AT-hook 2 \\
\hline ENSG00000101188 & 4,75 & $1,68 \mathrm{E}-51$ & NTSR1 & neurotensin receptor (high aff.) \\
\hline ENSG00000179918 & 4,76 & $2,44 \mathrm{E}-49$ & SEPHS2 & selenophosphate synthetase 2 \\
\hline ENSG00000179886 & 9,29 & $3,26 \mathrm{E}-49$ & TIGD5 & tigger transposable element der.5 \\
\hline ENSG00000174564 & 19,57 & $4,95 \mathrm{E}-48$ & IL20RB & interleukin 20 receptor beta \\
\hline ENSG00000225614 & 10,65 & $9,66 \mathrm{E}-46$ & ZNF469 & zinc finger protein 469 \\
\hline ENSG00000139178 & 6,86 & $9,43 \mathrm{E}-44$ & C1RL & complement component 1 , \\
\hline ENSG00000185561 & 10,75 & $1,47 \mathrm{E}-43$ & TLCD2 & TLC domain containing 2 \\
\hline ENSG00000176678 & 8,90 & $6,09 \mathrm{E}-43$ & FOXL1 & forkhead box L1 \\
\hline ENSG00000265688 & 10,58 & $2,37 \mathrm{E}-41$ & MAFG-AS1 & MAFG antisense RNA 1 \\
\hline ENSG00000196922 & 15,54 & $3,01 \mathrm{E}-41$ & ZNF252P & zinc finger protein 252 pseudoge. \\
\hline ENSG00000163216 & 27,76 & $4,66 \mathrm{E}-40$ & SPRR2D & small proline-rich protein $2 \mathrm{D}$ \\
\hline \multicolumn{5}{|c|}{ B) HCT116-jou-Knockdown (siRNA) (Down-regulated genes) } \\
\hline ENSG00000142871 & 0,19 & $1,84 \mathrm{E}-60$ & CYR61 & cysteine-rich, angiogenic inducer \\
\hline ENSG00000186480 & 0,07 & $6,02 \mathrm{E}-59$ & INSIG1 & insulin induced gene 1 \\
\hline ENSG00000079459 & 0,20 & $9,36 \mathrm{E}-50$ & FDFT1 & farnesyl-diphosphate farnesyltr.1 \\
\hline ENSG00000145632 & 0,24 & $4,46 \mathrm{E}-49$ & PLK2 & polo-like kinase 2 \\
\hline ENSG00000114019 & 0,15 & $3,07 \mathrm{E}-48$ & AMOTL2 & angiomotin like 2 ] \\
\hline ENSG00000198911 & 0,26 & $4,69 \mathrm{E}-44$ & SREBF2 & sterol regul. element bind. TF 2 \\
\hline ENSG00000113161 & 0,26 & $6,68 \mathrm{E}-43$ & HMGCR & 3-hydroxy-3-methylglutaryl-CoA red \\
\hline ENSG00000011426 & 0,26 & $9,52 \mathrm{E}-42$ & ANLN & anillin, actin binding protein \\
\hline ENSG00000169991 & 0,25 & $1,49 \mathrm{E}-40$ & IFFO2 & intermediate filament family orphan 2 \\
\hline ENSG00000115963 & 0,23 & $1,29 \mathrm{E}-38$ & RND3 & Rho family GTPase 3 \\
\hline ENSG00000158164 & 0,17 & $2,48 \mathrm{E}-38$ & TMSB15A & thymosin beta $15 \mathrm{a}$ \\
\hline ENSG00000099860 & 0,26 & $8,21 \mathrm{E}-37$ & GADD45B & growth arrest and DNA-damage \\
\hline ENSG00000130164 & 0,20 & $2,01 \mathrm{E}-36$ & LDLR & low density lipoprotein receptor \\
\hline ENSG00000052802 & 0,14 & $1,79 \mathrm{E}-35$ & MSMO1 & methylsterol monooxygenase 1 \\
\hline ENSG00000067064 & 0,27 & $3,24 \mathrm{E}-35$ & IDI1 & isopentenyl-diphosphate del iso 1 \\
\hline ENSG00000162772 & 0,29 & $5,52 \mathrm{E}-35$ & ATF3 & activating transcription factor 3 \\
\hline ENSG00000123975 & 0,30 & $1,56 \mathrm{E}-34$ & CKS2 & CDC28 protein kinase regul.sub2 \\
\hline ENSG00000185022 & 0,30 & $1,44 \mathrm{E}-33$ & MAFF & v-maf musculoaponeurotic fibros. \\
\hline ENSG00000147642 & 0,22 & $1,49 \mathrm{E}-33$ & SYBU & syntabulin (syntaxin-interacting) \\
\hline
\end{tabular}

$\begin{array}{lcccc}\text { C) HCT116-jou-Knockdown (siRNA) (KEGG enrichment pathways) } & & \\ \text { \#Term (KEGG pathway) } & \text { ID } & \text { Input number ackground numb } & \text { P-Value-corr } \\ \text { Metabolic pathways } & \text { hsa01100 } & 451 & 1243 & 3,20 \mathrm{E}-50 \\ \text { Ribosome } & \text { hsa03010 } & 99 & 138 & 5,66 \mathrm{E}-26 \\ \text { Spliceosome } & \text { hsa03040 } & 90 & 134 & 2,78 \mathrm{E}-22 \\ \text { Protein processing in endoplasmi, } & \text { hsa04141 } & 91 & 166 & 3,31 \mathrm{E}-18 \\ \text { Cell cycle } & \text { hsa04110 } & 72 & 124 & 2,03 \mathrm{E}-15 \\ \text { Pathways in cancer } & \text { hsa05200 } & 141 & 397 & 6,98 \mathrm{E}-15 \\ \text { Huntington's disease } & \text { hsa05016 } & 90 & 193 & 7,65 \mathrm{E}-15 \\ \text { Endocytosis } & \text { hsa04144 } & 105 & 260 & 5,18 \mathrm{E}-14 \\ \text { Alzheimer's disease } & \text { hsa05010 } & 80 & 168 & 1,10 \mathrm{E}-13 \\ \text { RNA transport } & \text { hsa03013 } & 81 & 172 & 1,10 \mathrm{E}-13 \\ \text { Ubiquitin mediated proteolysis } & \text { hsa04120 } & 69 & 137 & 8,81 \mathrm{E}-13 \\ \text { Epstein-Barr virus infection } & \text { hsa05169 } & 80 & 204 & 2,41 \mathrm{E}-10 \\ \text { Oocyte meiosis } & \text { hsa04114 } & 59 & 123 & 2,41 \mathrm{E}-10 \\ \text { Parkinson's disease } & \text { hsa05012 } & 64 & 142 & 2,51 \mathrm{E}-10 \\ \text { Apoptosis } & \mathrm{hsa} 04210 & 63 & 140 & 3,55 \mathrm{E}-10 \\ \text { Oxidative phosphorylation } & \text { hsa00190 } & 61 & 133 & 3,75 \mathrm{E}-10 \\ \text { Carbon metabolism } & \text { hsa01200 } & 55 & 113 & 5,90 \mathrm{E}-10 \\ \text { HTLV-I infection } & \text { hsa05166 } & 91 & 259 & 8,16 \mathrm{E}-10 \\ \text { MAPK signaling pathway } & \text { hsa04010 } & 90 & 255 & 8,16 \mathrm{E}-10\end{array}$


Suppl. Figure 1)

A) (overexpression)

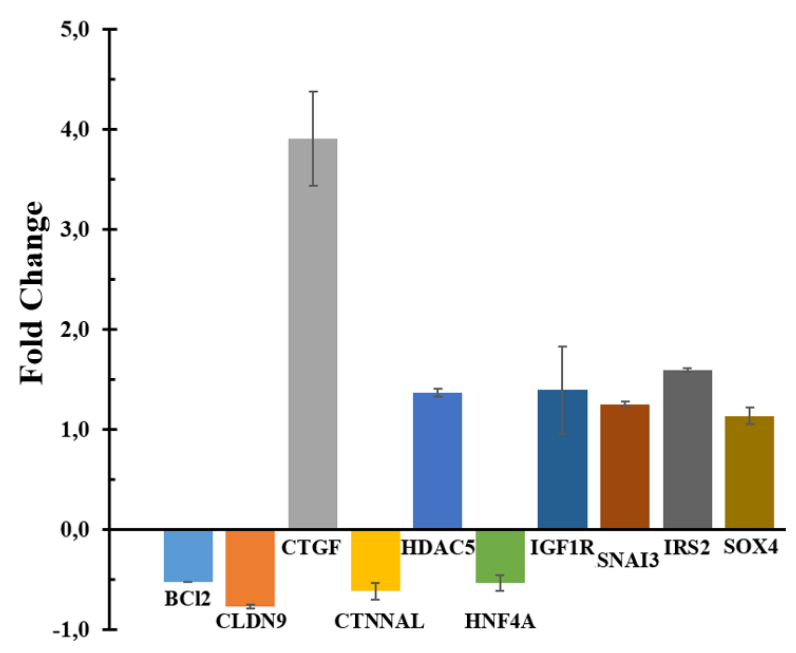

El Khoury et al.,

\section{B) Knock-down (siRNA)}

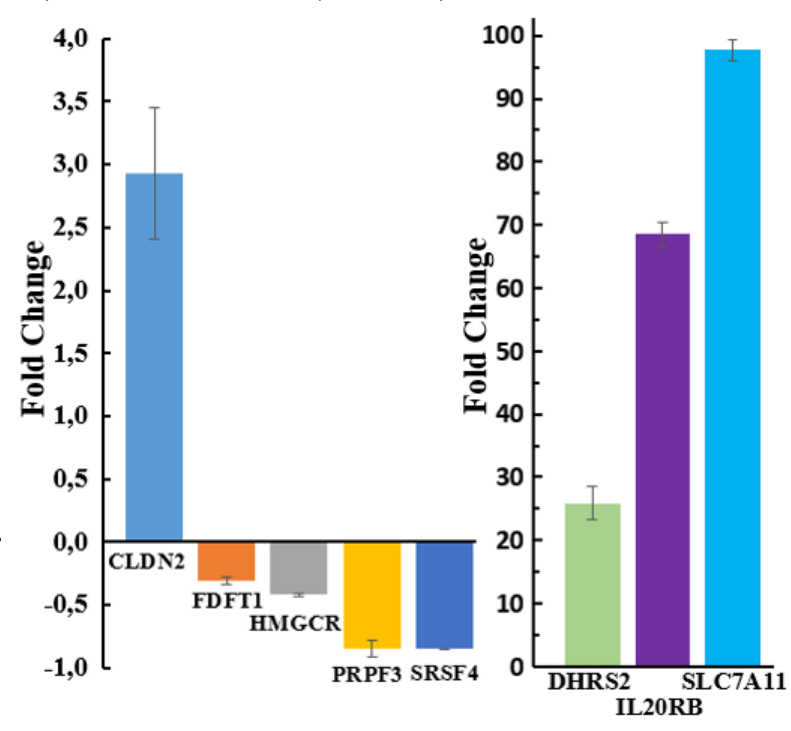

